\title{
A TOPOLOGICAL PROPERTY OF ASYMPTOTICALLY CONICAL SELF-SHRINKERS OF SMALL ENTROPY
}

\author{
JACOB BERNSTEIN AND LU WANG
}

\begin{abstract}
For any asymptotically conical self-shrinker with entropy less than or equal to that of a cylinder we show that the link of the asymptotic cone must separate the unit sphere into exactly two connected components, both diffeomorphic to the self-shrinker. Combining this with recent work of Brendle, we conclude that the round sphere uniquely minimizes the entropy among all non-flat two-dimensional self-shrinkers. This confirms a conjecture of Colding-Ilmanen-Minicozzi-White in dimension two.
\end{abstract}

\section{INTRODUCTION}

A hypersurface $\Sigma \subset \mathbb{R}^{n+1}$ is said to be a self-shrinker if it satisfies

$$
\mathbf{H}_{\Sigma}+\frac{\mathbf{x}^{\perp}}{2}=\mathbf{0} .
$$

Here $\mathbf{H}_{\Sigma}=-H_{\Sigma} \mathbf{n}_{\Sigma}=\Delta_{\Sigma} \mathbf{x}$ is the mean curvature vector of $\Sigma$ and $\mathbf{x}^{\perp}$ is the normal component of the position vector. Self-shrinkers arise naturally in the mean curvature flow as the time slices of solutions that move self-similarly by scaling. Specifically, if $\Sigma$ is a self-shrinker, then

$$
\left\{\Sigma_{t}\right\}_{t \in(-\infty, 0)}=\{\sqrt{-t} \Sigma\}_{t \in(-\infty, 0)}
$$

is a smooth mean curvature flow. Such self-similar flows are important as they model singularities of the mean curvature flow. From a variational point of view, self-shrinkers arise as critical points of the Gaussian (hyper)-surface area

$$
F[\Sigma]=\int_{\Sigma} \Phi(\mathbf{x}) d \mathcal{H}^{n}=(4 \pi)^{-\frac{n}{2}} \int_{\Sigma} e^{-\frac{|\mathbf{x}|^{2}}{4}} d \mathcal{H}^{n} .
$$

Here $\mathcal{H}^{n}$ is $n$-dimensional Hausdorff measure and $\Phi$ is the Gaussian normalized so that $F\left[\mathbb{R}^{n} \times\{0\}\right]=1$. Following Colding-Minicozzi [10], the entropy of a hypersurface is defined by

$$
\lambda[\Sigma]=\sup _{(\mathbf{y}, \rho) \in \mathbb{R}^{n+1} \times \mathbb{R}^{+}} F[\rho \Sigma+\mathbf{y}] .
$$

The Gaussian surface area and the entropy of self-shrinkers agree and so $\lambda\left[\mathbb{R}^{n} \times\{0\}\right]=1$.

A hypersurface, $\Sigma$, is asymptotically conical, if it is smoothly asymptotic to a regular cone; i.e., $\lim _{\rho \rightarrow 0} \rho \Sigma=\mathcal{C}(\Sigma)$ in $C_{\text {loc }}^{\infty}\left(\mathbb{R}^{n+1} \backslash\{\mathbf{0}\}\right)$ for $\mathcal{C}(\Sigma)$ a regular cone. Our main result is a topological restriction on asymptotically conical self-shrinkers with small entropy:

Theorem 1.1. Let $\Sigma$ be an asymptotically conical self-shrinker in $\mathbb{R}^{n+1}$ for $n \geq 2$. If $\lambda[\Sigma] \leq \lambda\left[\mathbb{S}_{*}^{n-1}\right]$, then $\mathcal{L}(\Sigma)$, the link of the asymptotic cone $\mathcal{C}(\Sigma)$, separates $\mathbb{S}^{n}$ into two connected components both diffeomorphic to $\Sigma$. As a consequence, $\mathcal{L}(\Sigma)$ is connected.

The first author was partially supported by the NSF Grant DMS-1307953. The second author was partially supported by the Chapman Fellowship of the Imperial College London and by the AMS-Simons Travel Grant 2012-2014 and the NSF Grant DMS-1406240. 
Here $\mathbb{S}^{n} \subset \mathbb{R}^{n+1}$ is the unit sphere centered at the origin and, for $0 \leq k \leq n$,

$$
\mathbb{S}_{*}^{n-k} \times \mathbb{R}^{k}=\left\{(\mathbf{x}, \mathbf{y}) \in \mathbb{R}^{n-k+1} \times \mathbb{R}^{k}=\mathbb{R}^{n+1}:|\mathbf{x}|^{2}=2(n-k)\right\}
$$

are the maximally symmetric self-shrinking cylinders with $k$-dimensional sping 1 As the $\mathbb{S}_{*}^{n-k} \times \mathbb{R}^{k}$ are self-shrinkers, their Gaussian surface area and entropy agree. That is,

$$
\lambda_{n}=\lambda\left[\mathbb{S}^{n}\right]=F\left[\mathbb{S}_{*}^{n}\right]=F\left[\mathbb{S}_{*}^{n} \times \mathbb{R}^{l}\right] .
$$

By a computation of Stone [24],

$$
2>\lambda_{1}>\frac{3}{2}>\lambda_{2}>\ldots>\lambda_{n}>\ldots \rightarrow \sqrt{2} .
$$

When $n=2$, Theorem 1.1 implies that $\Sigma$ is diffeomorphic to an open disk. This allows us to completely classify self-shrinkers in $\mathbb{R}^{3}$ of small entropy. Indeed, combining the work of Colding-Ilmanen-Minicozzi-White [9] (see also [2]) and Brendle [4] with Theorem 1.1. we conclude:

Corollary 1.2. If $\Sigma \subset \mathbb{R}^{3}$ is a self-shrinker with $\lambda[\Sigma] \leq \lambda_{1}$, then $\Sigma$ is either $\mathbb{S}_{*}^{2}$, or some rotation of $\mathbb{R}^{2} \times\{0\}$ or $\mathbb{S}_{*}^{1} \times \mathbb{R}$. In fact, there is a $\delta_{0}>0$ so that these are the only self-shrinkers with entropy less than $\lambda_{1}+\delta_{0}$.

This answers affirmatively the $n=2$ case of [9. Conjecture 0.10] which asks whether $\mathbb{S}_{*}^{n}$ minimizes the entropy amongst all non-flat self-shrinkers. In addition, this gives, for mean curvature flows in $\mathbb{R}^{3}$, that $\lambda_{2}$ is the best constant in White's formulation [26] of Brakke's regularity theorem [3]. Another simple consequence of Corollary 1.2 is a sharp lower bound for the entropy of all closed surfaces in $\mathbb{R}^{3}$ (see [2. Theorem 1.1] for a different approach which works for all $2 \leq n \leq 6$ ) and a new entropy lower bound for all closed surfaces in $\mathbb{R}^{3}$ of positive genus:

Corollary 1.3. If $\Sigma$ is a closed surface in $\mathbb{R}^{3}$, then $\lambda[\Sigma] \geq \lambda_{2}$ with equality if and only if $\Sigma$ is, up to translations and scalings, $\mathbb{S}_{*}^{2}$. Furthermore, if $\Sigma$ has positive genus, then $\lambda[\Sigma]>\lambda_{1}$.

Our proof of Theorem 1.1 parallels that of Colding-Ilmanen-Minicozzi-White, who, in [9], showed that $\mathbb{S}_{*}^{n}$ uniquely minimizes the entropy within the class of closed selfshrinkers. Indeed, our arguments may be thought of as a natural analog, in the asymptotically conical setting, of their arguments. However, we wish to emphasize one crucial difference between our proofs. Namely, in [9] the authors exploit the fact that a closed hypersurface must form a singularity in finite time - indeed, they point out this is the main obstruction to extending their result to the non-compact setting. We, however, make use of the fact that flows of asympotically conical surfaces of small entropy must exist without singularities for long-time to show that the flows eventually become star-shaped with respect to the origin.

More precisely, inspired by [9], we first study properties of a quantity along a smooth mean curvature flow that we call the shrinker mean curvature - see (3.1). In particular, we use a parabolic maximum principle on non-compact manifolds as in [12] to show that if the initial surface of a smooth mean curvature flow is well-behaved at infinity and has positive shrinker mean curvature, then the shrinker mean curvature remains positive along the flow - see Proposition 3.2. We also use a standard parabolic maximum principle to conclude

\footnotetext{
${ }^{1}$ In $\mathbb{R}^{n+1}$ a round sphere is a self-shrinker if and only if it is centered at the origin and its radius is $\sqrt{2 n}$. In our notation, the radii of $\mathbb{S}_{*}^{n}$ and $\mathbb{S}^{n}$ are, respectively, $\sqrt{2 n}$ and 1 . Thus, $\mathbb{S}_{*}^{n}$ is a self-shrinker while $\mathbb{S}^{n}$ is not.
} 
that for such a flow, the shrinker mean curvature controls the second fundamental form see Proposition 3.3 .

A variant on the shrinker mean curvature is also considered in [9] where the above properties are shown to hold for smooth mean curvature flows of closed hypersurfaces. However, in [9] the authors find it convenient to think of self-shrinkers as static points and so instead work with a certain rescaled mean curvature flow. As such, their quantity has a different, but related, form. While this may appear to be merely a technical distinction it actually holds the key to our proof. Indeed, the quantity we consider makes sense for smooth mean curvature flows which start close to a self-shrinker but that persist up to (and beyond) the singular time of the self-shrinker. By its construction, this cannot be true of the quantity considered in [9].

We next observe that if $\Sigma$ is any non-flat asymptotically conical self-shrinker, then there are asymptotically conical perturbations $\Gamma^{ \pm}$of $\Sigma$ on each side of $\Sigma$ which have positive shrinker mean curvature (relative to the correct orientation), strictly smaller entropy and which have the same asymptotic cone as that of $\Sigma-$ see Proposition 4.2. As in [9], these two hypersurfaces are found by considering the normal exponential graphs of small multiples of the lowest eigenfunction of the (self-shrinker) stability operator. Again the non-compactness introduces certain technical difficulties.

In our third step, we consider the smooth mean curvature flows with initial surfaces $\Gamma^{ \pm}$. Directly using arguments in [9], we prove that for non-compact flows of positive shrinker mean curvature if a singularity develops, then the Gaussian density of the flows at the singular point must be at least $\lambda_{n-1}$. Hence, if the entropy of $\Sigma$ is at most $\lambda_{n-1}$, then the flows starting from $\Gamma^{ \pm}$never develop singularities. We further show that each time slice of these flows is smoothly asymptotic to the same cone as that of $\Sigma$.

Finally, we observe that any long-time solution of the mean curvature flow with positive shrinker mean curvature and which is asymptotically conical must have a time-slice which is star-shaped - see Theorem 4.6 and Proposition 5.1. Theorem 1.1 is then an immediate consequence. The proofs of Corollaries 1.2 and 1.3 are straightforward.

\section{NOTATION}

Let $\mathbb{R}^{n+1}$ denote the standard $(n+1)$-dimensional Euclidean space. We denote by

$$
B_{R}\left(\mathbf{x}_{0}\right)=\left\{\mathbf{x} \in \mathbb{R}^{n+1}:\left|\mathbf{x}-\mathbf{x}_{0}\right|<R\right\}
$$

the open Euclidean ball of radius $R$ centered at $\mathbf{x}_{0}$. When the center is the origin $\mathbf{0}$, we will omit it. A hypersurface in $\mathbb{R}^{n+1}$ is a proper codimension one submanifold of $\mathbb{R}^{n+1}$. At times it will be convenient to distinguish between a point $p \in \Sigma$ and its position vector $\mathbf{x}(p) \in \mathbb{R}^{n+1}$. Given a set $\Omega \subset \mathbb{R}^{n+1}$ we define the $\rho$-tubular neighborhood of $\Omega$ to be the open set

$$
\mathcal{T}_{\rho}(\Omega)=\bigcup_{p \in \Omega} B_{\rho}(p) \subset \mathbb{R}^{n+1} .
$$

Any hypersurface is two-sided and hence orientable - see for instance [20]. For this reason, we will always orient a hypersurface, $\Sigma$, by a choice of unit normal

$$
\mathbf{n}_{\Sigma}: \Sigma \rightarrow \mathbb{S}^{n} \subset \mathbb{R}^{n+1}
$$

For such a $\Sigma$, we let $g_{\Sigma}$ denote the induced Riemannian metric, $A_{\Sigma}$ be the second fundamental form (with respect to the choice of unit normal $\mathbf{n}_{\Sigma}$ ) and $H_{\Sigma}$ the associated scalar mean curvature which we take to be the trace of $A_{\Sigma}$. Observe that the mean curvature vector $\mathbf{H}_{\Sigma}=-H_{\Sigma} \mathbf{n}_{\Sigma}$ is well-defined independent of choices of unit normal as is the norm 
$\left|A_{\Sigma}\right|$ of the second fundamental form. For any hypersurface, $\Sigma$, and $\rho \in \mathbb{R}^{+}$we have the natural smooth map

$$
\Psi_{\rho}: \Sigma \times(-\rho, \rho) \rightarrow \mathcal{T}_{\rho}(\Sigma)
$$

given by $\Psi_{\rho}(p, s)=\mathbf{x}(p)+s \mathbf{n}_{\Sigma}(p)$. We say $\mathcal{T}_{\rho}(\Sigma)$ is a regular tubular neighborhood if this map is a diffeomorphism.

We consider space-time, $\mathbb{R}^{n+1} \times \mathbb{R}$, to be the set of space-time points $X=(\mathrm{x}, t) \in$ $\mathbb{R}^{n+1} \times \mathbb{R}$. And let $O=(\mathbf{0}, 0)$ be the space-time origin. We will often focus on the subset of space-time consisting of space-time points with negative time which we denote by $\mathbb{R}^{n+1} \times \mathbb{R}^{-}$. Given $R, \tau>0$, let

$$
C_{R, \tau}\left(X_{0}\right)=\left\{(\mathbf{x}, t) \in \mathbb{R}^{n+1} \times \mathbb{R}: \mathbf{x} \in B_{R}\left(\mathbf{x}_{0}\right),\left|t-t_{0}\right|<\tau\right\}
$$

be the parabolic cylinder of radius $R$ and height $\tau$ centered at $X_{0}=\left(\mathbf{x}_{0}, t_{0}\right)$. It will also be convenient to consider the backward parabolic cylinder

$$
C_{R, \tau}^{-}\left(X_{0}\right)=C_{R, \tau}\left(X_{0}\right) \cap \mathbb{R}^{n+1} \times\left\{t<t_{0}\right\} .
$$

The parabolic boundary of a parabolic cylinder is defined to be

$$
\partial_{P} C_{R, \tau}\left(X_{0}\right)=\partial C_{R, \tau}\left(X_{0}\right) \backslash\left(B_{R}\left(\mathbf{x}_{0}\right) \times\left\{t=t_{0}+\tau\right\}\right),
$$

where $\partial$ is the topological boundary. Likewise, $\partial_{P} C_{R, \tau}^{-}\left(X_{0}\right)=\partial C_{R, \tau}^{-}\left(X_{0}\right) \cap \partial_{P} C_{R, \tau}\left(X_{0}\right)$.

A smooth mean curvature flow is a collection of hypersurfaces $\left\{\Sigma_{t}\right\}_{t \in I}$, where $I$ is some interval in $\mathbb{R}$ and so that there is a smooth map $F: M \times I \rightarrow \mathbb{R}^{n+1}$ so that for each $t \in I, F(\cdot, t): M \rightarrow \Sigma_{t} \subset \mathbb{R}^{n+1}$ is a parameterization of $\Sigma_{t}$ and so that

$$
\left(\frac{\partial}{\partial t} F(p, t)\right)^{\perp}=\mathbf{H}_{\Sigma_{t}}(F(p, t)) .
$$

We will always take the hypersurfaces $\Sigma_{t}$ in a smooth mean curvature flow to be oriented so that the unit normal is smooth in $t$. It is often convenient to consider the space-time track of a smooth mean curvature flow

$$
\mathcal{S}=\left\{(\mathbf{x}(p), t) \in \mathbb{R}^{n+1} \times \mathbb{R}: p \in \Sigma_{t}\right\},
$$

which is a smooth submanifold of space-time (with boundary if $I$ contains either of its endpoints) that is transverse to each constant time hyperplane. We will not distinguish between a smooth mean curvature flow $\left\{\Sigma_{t}\right\}_{t \in I}$ and its space-time track and so denote both by $\mathcal{S}$. Along the space-time track of a smooth mean curvature flow $\mathcal{S}$, let $\frac{d}{d t}$ be the smooth vector field given by

$$
\frac{d}{d t}=\frac{\partial}{\partial t}+\mathbf{H}_{\Sigma_{t}}
$$

It is not hard to see that this vector field is tangent to $\mathcal{S}$ and the position vector satisfies

$$
\frac{d}{d t} \mathbf{x}(p, t)=\mathbf{H}_{\Sigma_{t}}(p) .
$$

It is straightforward (and standard) to compute the evolution of various geometric quantities with respect to this vector field - see for instance [11, Appendix B].

For a set $\Omega \subset \mathbb{R}^{n+1}$, an $\mathbf{x} \in \mathbb{R}^{n+1}$ and a $\rho \in \mathbb{R}^{+}$, let

(1) $\Omega+\mathbf{x}=\left\{\mathbf{y} \in \mathbb{R}^{n+1}: \mathbf{y}-\mathbf{x} \in \Omega\right\}$, the translation of $\Omega$ by $\mathbf{x}$; and

(2) $\rho \Omega=\left\{\mathbf{y} \in \mathbb{R}^{n+1}: \rho^{-1} \mathbf{y} \in \Omega\right\}$, the scaling of $\Omega$ by $\rho$.

Similarly, for a set $\Omega \subset \mathbb{R}^{n+1} \times \mathbb{R}$ in space-time, an $X \in \mathbb{R}^{n+1} \times \mathbb{R}$ and a $\rho \in \mathbb{R}^{+}$, 
(1) $\Omega+X=\left\{Y \in \mathbb{R}^{n+1} \times \mathbb{R}: Y-X \in \Omega\right\}$, the space-time translation of $\Omega$ by $X$; and

(2) $\rho \Omega=\left\{(\mathbf{y}, t) \in \mathbb{R}^{n+1}:\left(\rho^{-1} \mathbf{y}, \rho^{-2} t\right) \in \Omega\right\}$, the parabolic scaling of $\Omega$ by $\rho$.

Clearly, if $\mathcal{S}$ is the space-time track of a smooth mean curvature flow, then so is $\rho \mathcal{S}+X$ for any $\rho \in \mathbb{R}^{+}$and $X \in \mathbb{R}^{n+1} \times \mathbb{R}$.

\section{SHRINKER MEAN-CONVEXITY}

Let $\mathcal{S}=\left\{\Sigma_{t}\right\}_{t \in[-1, T)}$ be a smooth mean curvature flow. Along the flow $\mathcal{S}$, we define the shrinker mean curvature relative to the space-time point $X_{0}=\left(\mathbf{x}_{0}, t_{0}\right)$ to be

$$
S_{\Sigma_{t}}^{X_{0}}(p)=2\left(t_{0}-t\right) H_{\Sigma_{t}}(p)-\left(\mathbf{x}(p)-\mathbf{x}_{0}\right) \cdot \mathbf{n}_{\Sigma_{t}}(p) .
$$

We emphasize that, due to the $t$ dependence, this quantity is defined for $\mathcal{S}$. Given a time $t \in \mathbb{R}$ and hypersurface $\Sigma$, the shrinker mean curvature of $\Sigma$ relative to the space-time point $X_{0}$ and time $t$ is defined to be

$$
S_{\Sigma}^{X_{0}, t}(p)=2\left(t_{0}-t\right) H_{\Sigma}(p)-\left(\mathbf{x}(p)-\mathbf{x}_{0}\right) \cdot \mathbf{n}_{\Sigma}(p) .
$$

We recall the following evolution equation for the shrinker mean curvature due to Smoczyk [23, Proposition 4].

Lemma 3.1. Along a mean curvature flow $\left\{\Sigma_{t}\right\}_{t \in I}$, the shrinker mean curvature relative to $X_{0}$ satisfies

$$
\frac{d}{d t} S_{\Sigma_{t}}^{X_{0}}=\Delta_{\Sigma_{t}} S_{\Sigma_{t}}^{X_{0}}+\left|A_{\Sigma_{t}}\right|^{2} S_{\Sigma_{t}}^{X_{0}} .
$$

Observe that $\mathcal{S}$ is self-similar with respect to parabolic rescalings about $X_{0}$ if and only if $S_{\Sigma_{t}}^{X_{0}} \equiv 0$. More generally, if one parabolically dilates $\mathcal{S}$ about $X_{0}$, then the vector field of the normal variation of this family at $\mathcal{S}$ is the shrinker mean curvature vector relative to $X_{0}$. Hence, as parabolic dilations are symmetries of the mean curvature flow, (3.3) may be viewed as the linearization of the mean curvature flow. In a similar fashion, the quantities $\mathbf{e}_{i} \cdot \mathbf{n}_{\Sigma_{+}}$used by Ecker-Huisken [12] are generated by spatial translations and so also satisfy (3.3). Likewise, the mean curvature $H_{\Sigma_{t}}$ which also satisfies (3.3) arises in this manner from temporal translations.

We now use the maximum principle of Ecker-Huisken [12, Corollary 1.1] on noncompact manifolds to show that if a smooth mean curvature flow $\left\{\Sigma_{t}\right\}_{t \in[-1, T)}$ satisfies that $\Sigma_{-1}$ is shrinker mean convex with respect to $X_{0}$, then this remains true for all $t \in(-1, T)$.

Proposition 3.2. Let $\mathcal{S}=\left\{\Sigma_{t}\right\}_{t \in[-1, T)}$ be a smooth mean curvature flow in $\mathbb{R}^{n+1}$ with finite entropy. Suppose that $\Sigma_{-1}$ satisfies

$$
S_{\Sigma_{-1}}^{X_{0}}(p) \geq c\left(1+|\mathbf{x}(p)|^{2}\right)^{-\alpha}
$$

for some $c>0$ and $\alpha \geq 0$, and that

$$
M=\sup _{t \in[-1, T)} \sup _{\Sigma_{t} \backslash B_{R}}\left|A_{\Sigma_{t}}\right|+\left|\nabla_{\Sigma_{t}} A_{\Sigma_{t}}\right|+\left|\nabla_{\Sigma_{t}}^{2} A_{\Sigma_{t}}\right|<\infty
$$

for some $R>0$. For all $(p, t) \in \mathcal{S}$,

$$
S_{\Sigma_{t}}^{X_{0}}(p) \geq c\left(1+|\mathbf{x}(p)|^{2}+2 n(t+1)\right)^{-\alpha} .
$$


Proof. Let

$$
\eta(\mathbf{x}, t)=1+|\mathbf{x}|^{2}+2 n(t+1) .
$$

By [12, Lemma 1.1] we have that

$$
\left(\frac{d}{d t}-\Delta_{\Sigma_{t}}\right) \eta=0
$$

and so

$$
\left(\frac{d}{d t}-\Delta_{\Sigma_{t}}\right) \eta^{\alpha}=-\alpha(\alpha-1)\left|\nabla_{\Sigma_{t}} \log \eta\right|^{2} \eta^{\alpha}
$$

Define

$$
u(p, t)=\eta^{\alpha}(\mathbf{x}(p), t) S_{\Sigma_{t}}^{X_{0}}(p) \quad \text { for }(p, t) \in \mathcal{S} .
$$

Then it follows from Lemma 3.1 and 3.8 that

$$
\left(\frac{d}{d t}-\Delta_{\Sigma_{t}}\right) u+2 \alpha \nabla_{\Sigma_{t}} \log \eta \cdot \nabla_{\Sigma_{t}} u=\left|A_{\Sigma_{t}}\right|^{2} u+\alpha(\alpha+1)\left|\nabla_{\Sigma_{t}} \log \eta\right|^{2} u .
$$

Notice that

$$
\left|\nabla_{\Sigma_{t}} \log \eta\right|(p, t)=\frac{2\left|\mathbf{x}(p)^{\top}\right|}{1+|\mathbf{x}(p)|^{2}+2 n(t+1)}<2,
$$

and by 3.5 and 3.11 we have that on $\mathcal{S} \backslash C_{R, T+1}(\mathbf{0},-1)$,

$$
\left|\frac{d u}{d t}\right|(p, t)+\sum_{i=0}^{2}\left|\nabla_{\Sigma_{t}}^{i} u\right|(p, t) \leq C\left(M, \alpha, X_{0}\right)\left(1+|\mathbf{x}(p)|^{2}+2 n(t+1)\right)^{\alpha+1} .
$$

Invoking (3.4) and finiteness of the entropy, Theorem A.1 implies, when $R=0$, that

$$
\inf _{p \in \Sigma_{t}} u(p, t) \geq \inf _{p \in \Sigma_{-1}} u(p,-1) \geq c,
$$

giving immediately 3.6.

We further adapt some ideas of [12] - specifically the proof of [12, Lemma 4.1] - to prove a relationship between the shrinker mean curvature and the second fundamental form for shrinker mean convex flows.

Proposition 3.3. Let $\mathcal{S}=\left\{\Sigma_{t}\right\}_{t \in[-1, T)}$ be a smooth mean curvature flow in $\mathbb{R}^{n+1}$ with finite entropy. Suppose that $\Sigma_{-1}$ satisfies

$$
S_{\Sigma_{-1}}^{X_{0}}(p) \geq c\left(1+|\mathbf{x}(p)|^{2}\right)^{-\alpha}
$$

for some $c>0$ and $\alpha \geq 0$, and that

$$
\tilde{M}=\max \left\{\sup _{t \in[-1, T)} \sup _{\Sigma_{t} \backslash B_{R}}\left|A_{\Sigma_{t}}\right|+\left|\nabla_{\Sigma_{t}} A_{\Sigma_{t}}\right|+\left|\nabla_{\Sigma_{t}}^{2} A_{\Sigma_{t}}\right|, \sup _{\Sigma_{-1}}\left|A_{\Sigma_{-1}}\right|\right\}<\infty
$$

for some $R>0$. Then for all $(p, t) \in \mathcal{S}$,

$$
\left|A_{\Sigma_{t}}(p)\right| \leq c^{-1} \tilde{M}\left(1+|\mathbf{x}(p)|^{2}+R^{2}+2 n(t+1)\right)^{\alpha} S_{\Sigma_{t}}^{X_{0}}(p) .
$$


Proof. First observe that, by Proposition 3.2, for all $(p, t) \in \mathcal{S}$,

$$
S_{\Sigma_{t}}^{X_{0}}(p) \geq c\left(1+|\mathbf{x}(p)|^{2}+2 n(t+1)\right)^{-\alpha} .
$$

Thus, in view of 3.16, it suffices to prove 3.17) on $\mathcal{S} \cap C_{R, T+1}^{-}(\mathbf{0}, T)$.

Let

$$
u(p, t)=\left|A_{\Sigma_{t}}(p)\right|^{2} v^{2}(p, t)=\left|A_{\Sigma_{t}}(p)\right|^{2}\left|S_{\Sigma_{t}}^{X_{0}}(p)\right|^{-2} .
$$

By [11, Appendix B, (B.9)],

$$
\left(\frac{d}{d t}-\Delta_{\Sigma_{t}}\right)\left|A_{\Sigma_{t}}\right|^{2} \leq-\left.2\left|\nabla_{\Sigma_{t}}\right| A_{\Sigma_{t}}\right|^{2}+2\left|A_{\Sigma_{t}}\right|^{4} .
$$

And by Lemma 3.1 .

$$
\left(\frac{d}{d t}-\Delta_{\Sigma_{t}}\right) v^{2}=-2\left|A_{\Sigma_{t}}\right|^{2} v^{2}-6\left|\nabla_{\Sigma_{t}} v\right|^{2}
$$

Thus, we compute the evolution equation of $u$ :

$$
\begin{aligned}
\left(\frac{d}{d t}-\Delta_{\Sigma_{t}}\right) u= & \left|A_{\Sigma_{t}}\right|^{2}\left(\frac{d}{d t}-\Delta_{\Sigma_{t}}\right) v^{2}-2 \nabla_{\Sigma_{t}} v^{2} \cdot \nabla_{\Sigma_{t}}\left|A_{\Sigma_{t}}\right|^{2} \\
& +v^{2}\left(\frac{d}{d t}-\Delta_{\Sigma_{t}}\right)\left|A_{\Sigma_{t}}\right|^{2} \\
& \leq-6\left|A_{\Sigma_{t}}\right|^{2}\left|\nabla_{\Sigma_{t}} v\right|^{2}-2 \nabla_{\Sigma_{t}} v^{2} \cdot \nabla_{\Sigma_{t}}\left|A_{\Sigma_{t}}\right|^{2} \\
& -\left.2\left|\nabla_{\Sigma_{t}}\right| A_{\Sigma_{t}}\right|^{2} v^{2} .
\end{aligned}
$$

Using Young's inequality, we obtain the estimate:

$$
\begin{aligned}
-2 \nabla_{\Sigma_{t}} v^{2} \cdot \nabla_{\Sigma_{t}}\left|A_{\Sigma_{t}}\right|^{2}= & -\frac{\nabla_{\Sigma_{t}} v^{2}}{v^{2}} \cdot \nabla_{\Sigma_{t}}\left(\left|A_{\Sigma_{t}}\right|^{2} v^{2}\right)+\frac{\left|A_{\Sigma_{t}}\right|^{2}\left|\nabla_{\Sigma_{t}} v^{2}\right|^{2}}{v^{2}} \\
& -\nabla_{\Sigma_{t}} v^{2} \cdot \nabla_{\Sigma_{t}}\left|A_{\Sigma_{t}}\right|^{2} \\
= & -\frac{\nabla_{\Sigma_{t}} v^{2}}{v^{2}} \cdot \nabla_{\Sigma_{t}} u+4\left|A_{\Sigma_{t}}\right|^{2}\left|\nabla_{\Sigma_{t}} v\right|^{2} \\
& -4 v\left|A_{\Sigma_{t}}\right| \nabla_{\Sigma_{t}} v \cdot \nabla_{\Sigma_{t}}\left|A_{\Sigma_{t}}\right| \\
\leq & -\frac{\nabla_{\Sigma_{t}} v^{2}}{v^{2}} \cdot \nabla_{\Sigma_{t}} u+6\left|A_{\Sigma_{t}}\right|^{2}\left|\nabla_{\Sigma_{t}} v\right|^{2} \\
& +\left.2 v^{2}\left|\nabla_{\Sigma_{t}}\right| A_{\Sigma_{t}}\right|^{2}
\end{aligned}
$$

Hence,

$$
\left(\frac{d}{d t}-\Delta_{\Sigma_{t}}\right) u \leq-\frac{\nabla_{\Sigma_{t}} v^{2}}{v^{2}} \cdot \nabla_{\Sigma_{t}} u=-2 \nabla_{\Sigma_{t}} \log v \cdot \nabla_{\Sigma_{t}} u .
$$

Observe that the hypothesis 3.16 and 3.18 imply that

$$
\sup _{\mathcal{S} \cap \partial_{P} C_{R, t+1}^{-}(\mathbf{0}, t)} u \leq c^{-2} \tilde{M}^{2}\left(1+R^{2}+2 n(t+1)\right)^{2 \alpha} .
$$

Therefore the standard parabolic maximum principle implies that

$$
\sup _{\mathcal{S} \cap C_{R, t+1}^{-}(\mathbf{0}, t)} u \leq c^{-2} \tilde{M}^{2}\left(1+R^{2}+2 n(t+1)\right)^{2 \alpha},
$$

which proves the result. 


\section{SELF-SHRINKERS OF SMALL ENTROPY ASYMPTOTIC TO REGULAR CONES}

We define $\mathcal{A C S}_{n}$ to be the space of connected asymptotically conical self-shrinkers in $\mathbb{R}^{n+1}$. Observe that the hyperplanes through the origin are contained in $\mathcal{A C S} \mathcal{S}_{n}$. We denote by $\mathcal{A C S}_{n}^{*}$ the subspace of non-flat elements of $\mathcal{A C S}_{n}$. Furthermore, given a $\lambda \geq 1$, we let $\mathcal{A C S}_{n}[\lambda]$ be the set of elements of $\mathcal{A C S}_{n}$ with Gaussian surface area (and hence entropy) less than or equal to $\lambda$ and define $\mathcal{A C S}_{n}^{*}[\lambda]$ likewise.

The goal of this section is to prove the long-time existence of a smooth mean curvature flow starting from a perturbation of any element of $\mathcal{A C S}_{n}^{*}\left[\lambda_{n-1}\right]$. First we need the following properties of the lowest eigenfunction of the stability operator.

Proposition 4.1. Given $\Sigma \in \mathcal{A C S}_{n}^{*}$, there is a $\mu=\mu(\Sigma)<-1$ and a unique positive smooth function $f$ on $\Sigma$ which satisfies

(4.1) $L_{\Sigma} f=\Delta_{\Sigma} f-\frac{\mathbf{x}}{2} \cdot \nabla_{\Sigma} f+\left|A_{\Sigma}\right|^{2} f+\frac{1}{2} f=-\mu f \quad$ with $\quad \int_{\Sigma} f^{2} e^{-\frac{|\mathbf{x}|^{2}}{4}} d \mathcal{H}^{n}=1$.

Moreover, for each $\beta>0$, there are constants $C_{0}, \ldots, C_{m}, \ldots>1$ depending on $\Sigma$ and $\beta$ so that

$$
\begin{gathered}
C_{0}^{-1}\left(1+|\mathbf{x}(p)|^{2}\right)^{\frac{1}{2}+\mu-\beta}<f(p)<C_{0}\left(1+|\mathbf{x}(p)|^{2}\right)^{\frac{1}{2}+\mu+\beta}, \quad \text { and } \\
\\
\left|\nabla_{\Sigma}^{m} f(p)\right|<C_{m}\left(1+|\mathbf{x}(p)|^{2}\right)^{\frac{1}{2}+\mu+\beta-\frac{m}{2}} \quad \text { for all } m \geq 1
\end{gathered}
$$

Proof. Given functions $\phi, \psi$ on $\Sigma$, we define

$$
(\phi, \psi)_{0}=\int_{\Sigma} \phi \psi e^{-\frac{|\mathbf{x}|^{2}}{4}} d \mathcal{H}^{n}, \quad(\phi, \psi)_{1}=(\phi, \psi)_{0}+\left(\nabla_{\Sigma} \phi, \nabla_{\Sigma} \psi\right)_{0},
$$

and $\|\phi\|_{i}^{2}=(\phi, \phi)_{i}$ for $i=0,1$. Let $L_{w}^{2}(\Sigma)$ be the Hilbert space of functions on $\Sigma$ with finite $\|\cdot\|_{0}$-norm and $H_{w}^{1}(\Sigma)$ be the Hilbert space given by completing $C_{c}^{\infty}(\Sigma)$ using $\|\cdot\|_{1}$. Observe that $H_{w}^{1}(\Sigma)$ may be naturally identified with a subspace of $L_{w}^{2}(\Sigma)$. In fact, this embedding is compact - see Appendix B or [7, Theorem 3].

Denote by $B_{R}^{\Sigma}$ the intrinsic geodesic open ball in $\Sigma$ centered at a fixed point $p \in \Sigma$ with radius $R$. Note that $\bigcup_{R>0} B_{R}^{\Sigma}=\Sigma$ as $\Sigma$ is connected. As observed in [10, Remark 5.17], there is a $\mu_{R}=\mu_{R}(\Sigma)$ and a unique positive function $f_{R} \in H_{w}^{1}\left(\overline{B_{R}^{\Sigma}}\right)$ so that $L_{\Sigma} f_{R}+\mu_{R} f_{R}=0$ with $\left\|f_{R}\right\|_{0}^{2}=1$. Moreover, $\mu_{R}$ is characterized by

$$
\mu_{R}=\inf _{\phi} \frac{\int_{\Sigma}\left(\left|\nabla_{\Sigma} \phi\right|^{2}-\left|A_{\Sigma}\right|^{2} \phi^{2}-\frac{1}{2} \phi^{2}\right) e^{-\frac{|\mathbf{x}|^{2}}{4}} d \mathcal{H}^{n}}{\int_{\Sigma} \phi^{2} e^{-\frac{|\mathbf{x}|^{2}}{4}} d \mathcal{H}^{n}},
$$

where the infimum is taken over all smooth non-zero functions compactly supported in $B_{R}^{\Sigma}$. As $\Sigma \in \mathcal{A C S}_{n}^{*}$, it follows from [25, Lemma 2.1] that

$$
\left(1+|\mathbf{x}(p)|^{2}\right)\left|A_{\Sigma}(p)\right|^{2}=O(1)
$$

Obviously, $\mu_{R}$ is non-increasing in $R$. Thus, $\mu_{R} \rightarrow \mu$ as $R \rightarrow \infty$ for some $\mu>-\infty$. This further implies that $\left\|f_{R}\right\|_{1}^{2}$ is uniformly bounded. Hence, there is a sequence $R_{j} \rightarrow \infty$ so that $f_{R_{j}}$ converges to a function $f$ on $\Sigma$ weakly in $H_{w}^{1}$ and strongly in $L_{w}^{2}$. Clearly, $f \in H_{w}^{1}(\Sigma)$ satisfying that $L_{\Sigma} f+\mu f=0$ with $\|f\|_{0}^{2}=1$. The smoothness of $f$ follows from a standard elliptic regularity theory. As each $f_{R_{j}}$ is non-negative, so is $f$ and, in fact, $f>0$ on $\Sigma$ by the Harnack inequality. Finally, it follows from [10, Lemma 9.25] that such an $f$ is unique. 
As $\Sigma$ is non-flat, the classification of mean convex self-shrinkers [10, Theorem 0.17] implies that $H_{\Sigma}$ must change sign. Hence, [10, Theorem 9.36] implies that $\mu<-1$. For $\beta>0$, define

$$
\bar{g}(\mathbf{x})=|\mathbf{x}|^{1+2 \mu+2 \beta} \quad \text { and } \quad \underline{g}(\mathbf{x})=|\mathbf{x}|^{1+2 \mu-2 \beta} .
$$

Then, invoking [10, Lemma 3.20] and 44.6, a direct computation gives

$$
L_{\Sigma} \bar{g}+\mu \bar{g}<0 \quad \text { and } \quad L_{\Sigma} \underline{g}+\mu \underline{g}>0 \quad \text { on } \Sigma \backslash \bar{B}_{\mathcal{R}},
$$

for some $\mathcal{R}=\mathcal{R}(\beta, \Sigma)>1$ sufficiently large. Next we choose $C>1$ so that

$$
C^{-1} \underline{g}<f<C \bar{g} \quad \text { on } \partial\left(\Sigma \backslash B_{\mathcal{R}}\right) .
$$

Consider the Dirichlet problem

$$
\begin{cases}L_{\Sigma} g+\mu g=0 & \text { on } \Sigma \cap\left(B_{i} \backslash \bar{B}_{\mathcal{R}}\right), \\ g=f & \text { on } \partial\left(\Sigma \backslash B_{\mathcal{R}}\right), \\ g=C \bar{g} & \text { on } \partial\left(\Sigma \backslash B_{i}\right) .\end{cases}
$$

Note that the zeroth order term of the differential equation in (4.10) has a negative sign. Thus, it follows from [14. Theorems 8.3 and 8.13 ] and the maximum principle that for each $i>\mathcal{R}$, the problem (4.10) has a unique smooth solution $g=g_{i}$ bounded between $C^{-1} g$ and $C \bar{g}$. Passing $i \rightarrow \infty$, by the Arzela-Ascoli theorem, $g_{i}$ converges in $C_{l o c}^{\infty}\left(\Sigma \backslash B_{\mathcal{R}}\right)$ to some function $\tilde{g}$ so that $L_{\Sigma} \tilde{g}+\mu \tilde{g}=0$ and $\tilde{g}=f$ on $\partial\left(\Sigma \backslash B_{\mathcal{R}}\right)$. Moreover,

$$
C^{-1}|\mathbf{x}(p)|^{1+2 \mu-2 \beta}<\tilde{g}(p)<C|\mathbf{x}(p)|^{1+2 \mu+2 \beta} .
$$

It follows from 4.6 and elliptic Schauder estimates that $\left|\nabla_{\Sigma} \tilde{g}\right|$ grows at most polynomially. This together with the Euclidean volume growth of $\Sigma$ implies that

$$
\int_{\Sigma \backslash B_{\mathcal{R}}}\left|\nabla_{\Sigma} \tilde{g}\right|^{2} e^{-\frac{|\mathbf{x}|^{2}}{4}} d \mathcal{H}^{n}<\infty .
$$

To conclude the proof, it suffices to show that $h=f-\tilde{g}=0$ on $\Sigma \backslash B_{\mathcal{R}}$. Let $\phi_{i}$ be a cut-off function on $\mathbb{R}^{n+1}$ so that $\phi_{i}=1$ in $B_{i}, \phi_{i}=0$ outside $B_{2 i}$, and $\left|D \phi_{i}\right|<2 / i$. Since $L_{\Sigma} h+\mu h=0$ with $h=0$ on $\partial\left(\Sigma \backslash B_{\mathcal{R}}\right)$, multiplying both sides of the equation by $h \phi_{i}^{2} e^{-|\mathbf{x}|^{2} / 4}$ and integration by parts give that

$$
\begin{aligned}
\int_{\Sigma \backslash B_{\mathcal{R}}}\left|\nabla_{\Sigma} h\right|^{2} \phi_{i}^{2} e^{-\frac{|\mathbf{x}|^{2}}{4}} d \mathcal{H}^{n} & -\int_{\Sigma \backslash B_{\mathcal{R}}}\left(\left|A_{\Sigma}\right|^{2}+\frac{1}{2}+\mu\right) h^{2} \phi_{i}^{2} e^{-\frac{|\mathbf{x}|^{2}}{4}} d \mathcal{H}^{n} \\
& +\int_{\Sigma \cap\left(B_{2 i} \backslash B_{i}\right)} 2 h \phi_{i} \nabla_{\Sigma} \phi_{i} \cdot \nabla_{\Sigma} h e^{-\frac{|\mathbf{x}|^{2}}{4}} d \mathcal{H}^{n}=0 .
\end{aligned}
$$

Thus, sending $i \rightarrow \infty$ and invoking that $\mu<-1$, the monotone convergence theorem together with (4.11) and (4.12) implies that $h=0$ on $\Sigma \backslash B_{\mathcal{R}}$ and so (4.2) follows.

Finally, letting $\Sigma_{t}=\sqrt{-t} \Sigma$, we define

$$
\tilde{f}(q, t)=(-t)^{\mu+\frac{1}{2}} f\left(\frac{q}{\sqrt{-t}}\right) \quad \text { for } t<0 \text { and } q \in \Sigma_{t} .
$$

Then (4.1) implies that

$$
\left(\frac{d}{d t}-\Delta_{\Sigma_{t}}\right) \tilde{f}=\left|A_{\Sigma_{t}}\right|^{2} \tilde{f}
$$

And (4.2) gives that, if $t \in[-1,0)$ and $q \in \Sigma_{t} \cap\left(B_{2} \backslash B_{1}\right)$, then

$$
0<\tilde{f}(q, t)<C^{\prime}(\Sigma, \mu, \beta)(-t)^{-\beta} \text {. }
$$


Recall that $\Sigma_{t} \rightarrow \mathcal{C}(\Sigma)$ in $C_{\text {loc }}^{\infty}\left(\mathbb{R}^{n+1} \backslash\{\mathbf{0}\}\right)$ as $t \rightarrow 0$. Thus, it follows from standard parabolic regularity theory that for each integer $m \geq 1$, there is a $C^{\prime \prime}=C^{\prime \prime}\left(\Sigma, C^{\prime}, m\right)$ so that if $p \in \Sigma \backslash B_{R}$, then

$$
\left|\nabla_{\Sigma}^{m} f(p)\right| \leq C^{\prime \prime}|\mathbf{x}(p)|^{1+2 \mu+2 \beta-m}
$$

proving 4.3.

We next use the lowest eigenfunction of the stability operator to perturb any element of $\mathcal{A C S}_{n}^{*}$ in order to strictly decrease its entropy.

Proposition 4.2. Let $\Sigma \in \mathcal{A C S}_{n}^{*}$ and let $C_{0}$, $\mu$, and $f$ be as in Proposition 4.1. There is an $\epsilon_{0}=\epsilon_{0}(\Sigma) \in(0,1)$ and $K=K(\Sigma)>1$ so that if $|\epsilon|<\epsilon_{0}$, then:

(1) the normal graph over $\Sigma$ given by

$$
\Gamma^{\epsilon}=\left\{\mathbf{y} \in \mathbb{R}^{n+1}: \mathbf{y}=\mathbf{x}(p)+\epsilon f(p) \mathbf{n}_{\Sigma}(p), p \in \Sigma\right\}
$$

is a smooth hypersurface;

(2) for all $R>1$ we have that $\Gamma^{\epsilon} \backslash B_{K R} \subset \mathcal{T}_{1 / R}(\mathcal{C}(\Sigma))$;

(3) $\Gamma^{\epsilon}$ is asymptotically conical and, in particular, given $\delta>0$ there is a $\kappa \in(0,1)$ and $\mathcal{R}>1$ depending only on $\delta$ and $\Sigma$ so that if $p \in \Sigma \backslash B_{\mathcal{R}}$ and $r=\kappa|\mathbf{x}(p)|$, then $\Gamma^{\epsilon} \cap B_{r}(p)$ can be written as a connected graph of a function $w$ over $T_{p} \Sigma$ with $|D w| \leq \delta$;

(4) by a suitable choice of the normal to $\Gamma^{\epsilon}$,

$$
S_{\Gamma^{\epsilon}}^{O,-1}(p) \geq-\mu|\epsilon| C_{0}^{-1}\left(1+|\mathbf{x}(p)|^{2}\right)^{\mu} \geq 0
$$

(5) if $\epsilon \neq 0$, then $\lambda\left[\Gamma^{\epsilon}\right]<\lambda[\Sigma]$.

Proof. By Proposition 4.1 $\mu<-1$, and so if $\beta=\frac{1}{2} \min \{1,-\mu-1\}$, then $0<\beta \leq 1 / 2$ and $\mu+\beta<-1$. As $\mu$ depends only on $\Sigma$, so does $\beta$. Thus the constants $C_{0}, C_{1}$ in Proposition 4.1 depend only on $\Sigma$ as well. Now define the mapping

$$
\Xi_{\epsilon}: \Sigma \rightarrow \Gamma^{\epsilon} \subset \mathbb{R}^{n+1}, \quad \Xi_{\epsilon}(p)=\mathbf{x}(p)+\epsilon f(p) \mathbf{n}_{\Sigma}(p)
$$

As $\Sigma \in \mathcal{A C S}_{n}^{*}$, in view of [25, Lemma 2.2], there is a $\rho>0$ so that $\mathcal{T}_{\rho}(\Sigma)$ is a regular tubular neighborhood. By Proposition 4.1. $f$ is smooth and uniformly bounded by $C_{0}$ and so, if $|\epsilon|<\rho / C_{0}$, then $\Xi_{\epsilon}(p)=\Psi_{\rho}(p, \epsilon f(p))$ and so $\Gamma^{\epsilon}$ is the image under the diffeomorphism $\Psi_{\rho}$ of the graph of $\epsilon f$ in $\Sigma \times(-\rho, \rho)$. In particular $\Gamma^{\epsilon}$ is a hypersurface, proving Item (1).

As $\mu+\beta<-1$, Item (2) follows directly from (4.2) for sufficiently large $K=K(\Sigma)$.

Let $\delta>0$ be any given small constant and $\mathcal{R}=\mathcal{R}(\delta, \Sigma)$ be a sufficiently large constant which may change among lines. By [25. Lemma 2.2], there is a $\kappa=\kappa(\delta, \Sigma) \in(0,1)$ so that if $p \in \Sigma \backslash B_{\mathcal{R}}$ and $r=\kappa|\mathbf{x}(p)|$, then $\Sigma \cap B_{2 r}(p)$ is given by a connected graph of a function over $T_{p} \Sigma$ with gradient bounded by $\delta / 2$. As $\beta$ was chosen so $\beta+\mu<-1$, (4.2) and 4.3 together with [25, Lemma 2.1], and a direct computation (see the proof of [25. Lemma 2.4]) imply that if $q \in \Sigma \cap B_{2 r}(p)$, then

$$
\left|\mathbf{n}_{\Gamma^{\epsilon}}\left(\Xi_{\epsilon}(q)\right) \cdot \mathbf{n}_{\Sigma}(q)\right|>1-C \epsilon(1+|\mathbf{x}(p)|)^{-2}
$$

where $C>0$ depends only on $C_{0}, C_{1}$ and $\Sigma$. For $|\epsilon|<\rho / C_{0},\left|\Xi_{\epsilon}(p)-p\right|<\rho$ and so

$$
\Gamma^{\epsilon} \cap B_{r}(p) \subset \Xi_{\epsilon}\left(\Sigma \cap B_{2 r}(p)\right) \text {. }
$$

Combining 4.21) and 4.22, if $p \in \Sigma \backslash B_{\mathcal{R}}$, we conclude that $\Gamma^{\epsilon} \cap B_{r}(p)$ can be written as a connected graph of a function over $T_{p} \Sigma$ with gradient bounded by $\delta$. In view of 4.2. 
and (4.3), it follows from Item (2) that $\Gamma^{\epsilon}$ is smoothly asymptotic to $\mathcal{C}(\Sigma)$. This completes the proof of Item (3).

As computed in the proof of [25, Lemma 2.4], it follows from an appropriate choice of unit normal to $\Gamma^{\epsilon}$ and (4.1) that

$$
S_{\Gamma^{\epsilon}}^{O,-1}=2|\epsilon| L_{\Sigma} f+Q_{\Sigma}\left(\epsilon f, \epsilon \nabla_{\Sigma} f, \epsilon \nabla_{\Sigma}^{2} f\right)=-2|\epsilon| \mu f+Q_{\Sigma}\left(\epsilon f, \epsilon \nabla_{\Sigma} f, \epsilon \nabla_{\Sigma}^{2} f\right) .
$$

Where $Q_{\Sigma}$ is a polynomial all of whose terms are of order at least 2 and which has uniformly bounded coefficients. This, together with (4.2), (4.3) and the fact that $\beta \leq 1 / 2$, implies Item (4) for all $|\epsilon|$ sufficiently small.

Finally, Item (5) can be shown by modifying the arguments in the proof of [10, Theorem 0.15]. We leave the technical details to Appendix C.

We next use sphere barriers to show that smooth mean curvature flows which initially decay to a regular cone must continue to decay to the same cone for all positive time.

Lemma 4.3. Let $\left\{\Gamma_{t}\right\}_{t \in[-1, T)}$ be a smooth mean curvature flow in $\mathbb{R}^{n+1}$. Suppose that there is a regular cone, $\mathcal{C}$, and a $K>1$ so that $\Gamma_{-1} \backslash B_{K R} \subset \mathcal{T}_{1 / R}(\mathcal{C})$ for all $R>1$. Then there is a constant $K^{\prime}=K^{\prime}(\mathcal{C}, K)>1$ so that for all $R>1$ and $t \in[-1, T)$,

$$
\Gamma_{t} \backslash B_{K^{\prime}(R+\sqrt{2 n(t+1)})} \subset \mathcal{T}_{K^{\prime}(1+2 n(t+1)) R^{-1}}(\mathcal{C}) .
$$

Proof. For $\mathbf{x} \in \mathbb{R}^{n+1}$, let $\varrho(\mathbf{x})=\inf \left\{\rho \geq 0: B_{\rho}(\mathbf{x}) \cap\left(B_{K R} \cup \mathcal{T}_{1 / R}(\mathcal{C})\right) \neq \emptyset\right\}$. Define an open subset of $\mathbb{R}^{n+1}$ by

$$
U=\bigcup_{\mathbf{x} \in \mathbb{R}^{n+1}} B_{\varrho(\mathbf{x})}(\mathbf{x}) .
$$

Clearly, $U \cap \Gamma_{-1}=\emptyset$. For $t \geq-1$, set

$$
\begin{gathered}
\varrho(\mathbf{x}, t)=\left\{\begin{array}{cc}
\sqrt{\varrho(\mathbf{x})^{2}-2 n(t+1)} & \varrho(\mathbf{x})^{2} \geq 2 n(t+1) \\
0 & \varrho(\mathbf{x})^{2}<2 n(t+1),
\end{array}\right. \\
U_{t}=\bigcup_{\mathbf{x} \in \mathbb{R}^{n+1}} B_{\varrho(\mathbf{x}, t)}(\mathbf{x}) .
\end{gathered}
$$

By the avoidance principle for mean curvature flow, $U_{t} \cap \Gamma_{t}=\emptyset$ for all $t \in[-1, T)$.

Let $d_{\mathcal{C}}$ denote the distance to the cone $\mathcal{C}$. Observe that

$$
\varrho(\mathbf{x})=\max \left\{\min \left\{|\mathbf{x}|-K R, d_{\mathcal{C}}(\mathbf{x})-R^{-1}\right\}, 0\right\}
$$

and so the set $U_{t}^{+}=\left\{\mathbf{x} \in \mathbb{R}^{n+1}: \varrho(\mathbf{x}, t)>0\right\}$ satisfies

(4.29) $U_{t}^{+}=\left\{\mathbf{x} \in \mathbb{R}^{n+1}:|\mathbf{x}|>K R+\sqrt{2 n(t+1)}, d_{\mathcal{C}}(\mathbf{x})>R^{-1}+\sqrt{2 n(t+1)}\right\}$.

Clearly, $U_{t}^{+} \subset U_{t}$ and, in general, is a proper subset. Hence,

$$
\Gamma_{t} \backslash B_{K R+\sqrt{2 n(t+1)}} \subset \mathcal{T}_{R^{-1}+\sqrt{2 n(t+1)}}(\mathcal{C}) .
$$

To conclude, pick a unit normal $\mathbf{n}_{\mathcal{C}}$ to $\mathcal{C} \backslash\{\mathbf{0}\}$ so there is a smooth map

$$
\Psi: \mathcal{C} \backslash\{\mathbf{0}\} \times \mathbb{R} \rightarrow \mathbb{R}^{n+1}, \quad \Psi(p, l)=\mathbf{x}(p)+l \mathbf{n}_{\mathcal{C}}(p) .
$$

As $\mathcal{L}(\mathcal{C})$, the link of $\mathcal{C}$, is compact and smooth, there is a $\vartheta \in(0,1 / 2)$ so that if

$$
N=\{(p, l) \in \mathcal{C} \times \mathbb{R}:|l|<2 \vartheta|\mathbf{x}(p)|\},
$$

then $\left.\Psi\right|_{N}$ is a diffeomorphism onto its image. 
Fix $t>-1$ and consider the part of $U_{t}^{c}$ far from the origin. That is, set $K^{\prime}=1+$ $2 K \vartheta^{-1}>4 K$ and

$$
V_{t}=U_{t}^{c} \cap\left\{\mathbf{x} \in \mathbb{R}^{n+1}:|\mathbf{x}| \geq K^{\prime}(R+\sqrt{2 n(t+1)})\right\} .
$$

If $\mathbf{y} \in V_{t}$, then, by the definition of $U_{t}$ and the fact that $|\mathbf{y}| \geq K^{\prime} R>K R, d_{\mathcal{C}}(\mathbf{y}) \leq R^{-1}+$ $\sqrt{2 n(t+1)}$. Hence, there is a point $p \in \mathcal{C}$ so $d_{\mathcal{C}}(\mathbf{y})=|\mathbf{y}-\mathbf{x}(p)| \leq R^{-1}+\sqrt{2 n(t+1)}$. This implies that

$$
\begin{aligned}
|\mathbf{x}(p)| & \geq K^{\prime}(R+\sqrt{2 n(t+1)})-\sqrt{2 n(t+1)}-R^{-1} \\
& \geq\left(K^{\prime}-1\right)(R+\sqrt{2 n(t+1)}) \\
& =2 K \vartheta^{-1}(R+\sqrt{2 n(t+1)})
\end{aligned}
$$

where the second inequality used that $R>1$ and so $R>R^{-1}$. Hence,

$$
\frac{|\mathbf{y}-\mathbf{x}(p)|}{|\mathbf{x}(p)|} \leq \frac{R^{-1}+\sqrt{2 n(t+1)}}{2 K \vartheta^{-1}(R+\sqrt{2 n(t+1)})}<\vartheta
$$

where we used that $R>R^{-1}$ and that $K>1$.

Thus, $\mathbf{y} \in \Psi(N)$ and so, up to reversing the orientation of $\mathcal{C}, \mathbf{y}=\Psi\left(p, d_{\mathcal{C}}(\mathbf{y})\right)=$ $\mathbf{x}(p)+d_{\mathcal{C}}(\mathbf{y}) \mathbf{n}_{\mathcal{C}}(p)$. Set $\mathbf{y}_{0}=\mathbf{x}(p)+\vartheta|\mathbf{x}(p)| \mathbf{n}_{\mathcal{C}}(p)$. Observe that

$$
\left|\mathbf{y}_{0}\right|-\left(\vartheta|\mathbf{x}(p)|-R^{-1}\right)=\left(\sqrt{1+\vartheta^{2}}-\vartheta\right)|\mathbf{x}(p)|+R^{-1}>K R
$$

where we used that, by (4.34), $|\mathbf{x}(p)|>4 K R$ and $\sqrt{1+\vartheta^{2}}-\vartheta>1 / 3$. Thus,

$$
B_{\vartheta|\mathbf{x}(p)|-R^{-1}}\left(\mathbf{y}_{0}\right) \cap B_{K R}=\emptyset \quad \text { and so } \quad \varrho\left(\mathbf{y}_{0}\right)=\vartheta|\mathbf{x}(p)|-R^{-1} .
$$

As $\mathbf{y} \notin U_{t}$, we have $\mathbf{y} \notin B_{\rho\left(\mathbf{y}_{0}, t\right)}\left(\mathbf{y}_{0}\right)$. That is, $\rho\left(\mathbf{y}_{0}, t\right) \leq\left|\mathbf{y}-\mathbf{y}_{0}\right|=\vartheta|\mathbf{x}(p)|-d_{\mathcal{C}}(\mathbf{y})$. Hence,

$$
\begin{aligned}
d_{\mathcal{C}}(\mathbf{y}) & \leq \vartheta|\mathbf{x}(p)|-\sqrt{\left(\vartheta|\mathbf{x}(p)|-R^{-1}\right)^{2}-2 n(t+1)} \\
& =\frac{\vartheta^{2}|\mathbf{x}(p)|^{2}-\left(\vartheta|\mathbf{x}(p)|-R^{-1}\right)^{2}+2 n(t+1)}{\vartheta|\mathbf{x}(p)|+\sqrt{\left(\vartheta|\mathbf{x}(p)|-R^{-1}\right)^{2}-2 n(t+1)}} \\
& <\frac{2 R^{-1} \vartheta|\mathbf{x}(p)|-R^{-2}+2 n(t+1)}{\vartheta|\mathbf{x}(p)|} \\
& <\frac{2}{R}+\frac{2 n(t+1)}{\vartheta|\mathbf{x}(p)|}<\frac{2(1+2 n(t+1))}{\vartheta R},
\end{aligned}
$$

where we used $|\mathbf{x}(p)|>R$ for the last inequality. Therefore, as $\Gamma_{t} \subset U_{t}^{c}$ and $K^{\prime}>2 / \vartheta$,

$$
\Gamma_{t} \backslash B_{K^{\prime}(R+\sqrt{2 n(t+1)})} \subset \mathcal{T}_{K^{\prime}(1+2 n(t+1)) R^{-1}(\mathcal{C}),}
$$

from which the result follows.

In what follows, we establish the existence and uniqueness of a smooth shrinker mean convex mean curvature flow starting from $\Gamma^{\epsilon}$ at time -1 . Moreover, we show that each time slice of this flow is smoothly asymptotic to $\mathcal{C}(\Sigma)$ and if the flow exists smoothly beyond time 0 , then the time zero slice lies strictly on one side of $\mathcal{C}(\Sigma)$. 
Proposition 4.4. Let $\Sigma \in \mathcal{A C S}_{n}^{*}$ and let $\Gamma^{\epsilon}$ be as in Proposition 4.2 for $\epsilon \neq 0$. There is a $T=T\left(\Gamma^{\epsilon}\right) \in(-1, \infty]$, and a unique smooth mean curvature flow $\left\{\Gamma_{t}^{\epsilon}\right\}_{t \in[-1, T)}$ with $\Gamma_{-1}^{\epsilon}=\Gamma^{\epsilon}$. Furthermore, there is an increasing function $R(t)>1$ on $[-1, \infty)$ depending only on $\Gamma^{\epsilon}$ so that:

(1) for $t \in[-1, T), \Gamma_{t}^{\epsilon}$ is smoothly asymptotic to $\mathcal{C}(\Sigma)$ and, in particular, for $p \in$ $\Gamma_{t}^{\epsilon} \backslash B_{R(t)}$,

$$
|\mathbf{x}(p)|\left|A_{\Gamma_{t}^{\epsilon}}(p)\right|+|\mathbf{x}(p)|^{2}\left|\nabla_{\Gamma_{t}^{\epsilon}} A_{\Gamma_{t}^{\epsilon}}(p)\right|+|\mathbf{x}(p)|^{3}\left|\nabla_{\Gamma_{t}^{\epsilon}}^{2} A_{\Gamma_{t}^{\epsilon}}(p)\right|<\tilde{C}_{0},
$$

where $\tilde{C}_{0}$ depends only on $\Gamma^{\epsilon}$;

(2) if $T<\infty$, then

$$
\lim _{t \rightarrow T} \sup _{B_{R(t)} \cap \Gamma_{t}^{\epsilon}}\left|A_{\Gamma_{t}^{\epsilon}}\right|=\infty
$$

(3) for $t<\min \{0, T\}, \Gamma_{t}^{\epsilon} \backslash \bar{B}_{R(t)}$ is given by the normal graph of a function $f_{t}$ over a region $\Omega_{t} \subset \Sigma_{t}=\sqrt{-t} \Sigma$ with $\Sigma_{t} \backslash B_{2 R(t)} \subset \Omega_{t}$ which satisfies $f_{-1}=\left.\epsilon f\right|_{\Omega_{-1}}$,

$$
\begin{gathered}
\tilde{C}_{1}^{-1}|\epsilon|(1+|\mathbf{x}(p)|)^{2 \mu}<\left|f_{t}(p)\right|<\tilde{C}_{1}(1+|\mathbf{x}(p)|)^{-1}, \quad \text { and } \\
(1+|\mathbf{x}(p)|)^{2}\left|\nabla_{\Sigma_{t}} f_{t}(p)\right|+(1+|\mathbf{x}(p)|)^{3}\left|\nabla_{\Sigma_{t}}^{2} f_{t}(p)\right|<\tilde{C}_{1},
\end{gathered}
$$

where $\tilde{C}_{1}$ depends only on $\Sigma$;

(4) for $t<\min \{0, T\}, \Gamma_{t}^{\epsilon} \cap \Sigma_{t}=\emptyset$, and if $T>0$, then $\Gamma_{0}^{\epsilon} \cap \mathcal{C}(\Sigma)=\emptyset$;

(5) by a suitable choice of the normal to $\Gamma^{\epsilon}$,

$$
S_{\Gamma_{t}^{\epsilon}}^{O}(p) \geq-\mu|\epsilon| C_{0}^{-1}\left(1+|\mathbf{x}(p)|^{2}+2 n(t+1)\right)^{\mu}>0 .
$$

Proof. First, it follows from Items (2) and (3) of Proposition 4.2 that $\Gamma^{\epsilon}$ smoothly asymptotes to the same regular cone, $\mathcal{C}(\Sigma)$, as that of $\Sigma$. Thus, $\Gamma^{\epsilon}$ has uniformly bounded $C^{m}$ norm for all $m$ and $\mathcal{T}_{\rho}\left(\Gamma^{\epsilon}\right)$ is a regular tubular neighborhood of $\Gamma^{\epsilon}$ for some $\rho>0$. Arguing as in the proof of [13, Theorem 4.2], there exists a smooth mean curvature flow starting from $\Gamma^{\epsilon}$ for small positive time. Indeed, the construction ensures that each of these time slices can be expressed as a smooth normal graph over $\Gamma^{\epsilon}$ with small $L^{\infty}$ norm. Denote the maximal extension of this flow to a smooth mean curvature flow by $\left\{\Gamma_{t}^{\epsilon}\right\}_{t \in[-1, T)}$.

Invoking Item (2) of Proposition 4.2, it follows from Lemma 4.3 and [25, Lemma 2.2] that there exist increasing functions $R(t)>1$ and $C(t)>1$ on $[-1, \infty)$ depending only on $\Gamma^{\epsilon}$ so that for $t \in(-1, T)$ and $p \in \Gamma_{t}^{\epsilon} \backslash B_{R(t)}, d_{\Sigma}(\mathbf{x}(p)) \leq C(t)|\mathbf{x}(p)|^{-1}$, where $d_{\Sigma}$ is the distance to $\Sigma$. Furthermore, the pseudo-locality property of mean curvature flow (cf. [18, Theorem 1.5]) together with Item (3) of Proposition 4.2 (for $\delta>0$ sufficiently small) implies that each slice $\Gamma_{\tau}^{\epsilon} \cap B_{r}(q)$ is a connected graph of a function $w_{\tau}$ over $T_{q} \Sigma$ with $\left|D w_{\tau}\right| \leq 2 \delta$. Here, $\tau \leq t$ and

$$
q \in \Sigma \text { is such that }|\mathbf{x}(p)-\mathbf{x}(q)|=d_{\Sigma}(\mathbf{x}(p)) \text { and } r=\varsigma \kappa|\mathbf{x}(q)|,
$$

for some universal small constant $\varsigma$. Replacing the localization function in the proof of [11. Propositions 3.21 and 3.22] with $\left(1-|\mathbf{x}|^{2}\right)_{+}^{3}$, we conclude that, for $m \geq 0$,

$$
\sup _{\tau \in[-1, t]} \sup _{\Gamma_{\tau}^{\epsilon} \cap B_{\frac{r}{2}}(q)}\left|\nabla_{\Gamma_{\tau}^{\epsilon}}^{m} A_{\Gamma_{\tau}^{\epsilon}}\right| \leq C^{\prime}\left(m, \delta, \Gamma^{\epsilon}\right) r^{-m-1} .
$$

This, together with Item (2) of Proposition 4.2 and Lemma 4.3 , immediately gives Item (1) and allows us to invoke [6. Theorem 1.1] to establish that the flow $\left\{\Gamma_{t}^{\epsilon}\right\}_{t \in[-1, T)}$ is the unique smooth mean curvature flow starting from $\Gamma^{\epsilon}$ at time -1 . Hence, it remains only to verify Items (2), (3), (4) and (5). 
To show Item (2), we argue by contradiction. If $T<\infty$ and

$$
\sup _{t \in[-1, T)} \sup _{\Gamma_{t}^{\epsilon}}\left|A_{\Gamma_{t}^{\epsilon}}\right|<\infty,
$$

then the same holds true for all higher order derivatives of the second fundamental form by [13, Theorem 3.7]. As $\Gamma^{\epsilon}$ decays smoothly to $\mathcal{C}(\Sigma)$, it follows from Item (1) that $\Gamma_{t}^{\epsilon}$ for $t<T$ smoothly decays to $\mathcal{C}(\Sigma)$ in a uniform manner. Thus, $\Gamma_{t}^{\epsilon} \rightarrow \Gamma_{T}^{\epsilon}$ smoothly and $\Gamma_{T}^{\epsilon}$ is a hypersurface in $\mathbb{R}^{n+1}$ smoothly decaying to $\mathcal{C}(\Sigma)$. By our previous discussion, we could extend smoothly the flow $\left\{\Gamma_{t}^{\epsilon}\right\}_{t \in[-1, T)}$ to a larger time interval $\left[-1, T^{\prime}\right)$ for some $T^{\prime}>T$. This contradicts the maximality of $T$.

By [25, Lemma 2.2], for $\tau \leq t<\min \{0, T\}, p \in \Sigma_{\tau}$ and $q$ and $r$ as in (4.45), $\Sigma_{\tau} \cap B_{r}(q)$ is the connected graph of a function $\tilde{w}_{\tau}$ over $T_{q} \Sigma$ with $r^{m-1}\left|D^{m} \tilde{w}_{\tau}\right|$ uniformly bounded for $1 \leq m \leq 3$. Below, let $C^{\prime \prime}=C^{\prime \prime}\left(\delta, \Sigma, \Gamma^{\epsilon}\right)$ be a sufficiently large constant which may change among lines. In view of (4.2) and (4.3), it is straightforward to check that

$$
\sum_{m=0}^{2} r^{-1-2 \mu-2 \beta+m}\left\|D^{m} w_{-1}-D^{m} \tilde{w}_{-1}\right\|_{L^{\infty}} \leq C^{\prime \prime} .
$$

Thus, combining (4.46) and 4.48), it follows from the mean curvature flow equation that, as long as $\beta$ is small enough so that $\mu+\beta<-1$, for $t<\min \{0, T\}$,

$$
\sum_{m=0}^{2} r^{m+1}\left\|D^{m} w_{t}-D^{m} \tilde{w}_{t}\right\|_{L^{\infty}} \leq C^{\prime \prime}
$$

Hence, using similar reasoning as in the proof of [25, Lemma 2.3], one verifies all of Item (3) except the lower bound on $\left|f_{t}\right|$, which will be a consequence of our proof of Item (4).

Next, by symmetry, it suffices to show Item (4) for $\epsilon>0$ and so $f_{-1}=\epsilon f>0$. Let

$$
T_{0}=\sup \left\{t_{0}<\min \{0, T\}: \Gamma_{t}^{\epsilon} \cap \Sigma_{t}=\emptyset \text { for all } t<t_{0}\right\} .
$$

We will show that $T_{0}=\min \{0, T\}$, implying the first part of Item (4), i.e., $\Gamma_{t}^{\epsilon} \cap \Sigma_{t}=\emptyset$ when $t<\min \{0, T\}$. First, we prove that $\Gamma_{t}^{\epsilon}$ remains disjoint from $\Sigma_{t}$ for $t$ close to -1 . By continuity, there is a $\delta^{\prime}>0$ small so that $\Gamma_{t}^{\epsilon} \cap \Sigma_{t} \cap \bar{B}_{2 R(0)}=\emptyset$ for all $t<-1+\delta^{\prime}$. In particular, if $t<-1+\delta^{\prime}$, then $f_{t}$ restricted to $\Sigma_{t} \cap \partial B_{2 R(0)}$ is strictly positive. As both $\Gamma_{t}^{\epsilon}$ and $\Sigma_{t}$ move by mean curvature, the equation for $f_{t}$ is given by a perturbation of the linearized mean curvature flow equation and thus, invoking [25, Lemma 2.1] and Item (3), we get that

$$
\left(\frac{d}{d t}-\Delta_{\Sigma_{t}}\right) f_{t}=\mathbf{a} \cdot \nabla_{\Sigma_{t}} f_{t}+b f_{t}
$$

where $\mathbf{a}$ and $b$ are uniformly bounded and depend on $\Sigma, f_{t}, \nabla_{\Sigma_{t}} f_{t}$, and $\nabla_{\Sigma_{t}}^{2} f_{t}$. The derivation of 4.51) involves lengthy but tedious computations for which we refer the reader to [21. Lemma 2.5]. Define

$$
\tilde{f}_{t}(p)=f_{t}(p) \eta^{-\mu}(\mathbf{x}(p), t)=f_{t}(p)\left(1+|\mathbf{x}(p)|^{2}+2 n(t+1)\right)^{-\mu} .
$$

Then it is easy to deduce that

$$
\left(\frac{d}{d t}-\Delta_{\Sigma_{t}}\right) \tilde{f}_{t}=\tilde{\mathbf{a}} \cdot \nabla_{\Sigma_{t}} \tilde{f}_{t}+\tilde{b} \tilde{f}_{t}
$$

where

$$
\tilde{\mathbf{a}}=\mathbf{a}+2 \mu \nabla_{\Sigma_{t}} \log \eta \quad \text { and } \quad \tilde{b}=b+\mu(\mu-1)\left|\nabla_{\Sigma_{t}} \log \eta\right|^{2}+\mu \mathbf{a} \cdot \nabla_{\Sigma_{t}} \log \eta
$$


Thus, together with (4.2) (choosing $\beta=1 / 2$ ), it follows from Theorem A.1 that, for $t<-1+\delta^{\prime}, \tilde{f}_{t}$ in $\Sigma_{t} \backslash B_{2 R(0)}$ is bounded from below by a positive constant. In particular, this implies that $T_{0} \geq-1+\delta^{\prime}$.

Assume that $T_{0}<\min \{0, T\}$. Invoking Item (3) and [25. Lemma 2.1] again, it follows from the parabolic Harnack inequality [19. Corollary 7.42] that $\tilde{f}_{t}$ restricted on $\Sigma_{t} \backslash B_{4 R(0)}$ for $t \leq T_{0}$ is also bounded from below by a positive constant. Thus, by the parabolic maximum principle on bounded domains, we conclude that $\Gamma_{T_{0}}^{\epsilon} \cap \Sigma_{T_{0}}=\emptyset$. Hence, we repeat the arguments in the previous paragraph to see that $\Gamma_{t}^{\epsilon} \cap \Sigma_{t}=\emptyset$ for $t \in\left[T_{0}, T_{0}^{\prime}\right]$ and $T_{0}^{\prime}>T_{0}$. This contradicts the definition of $T_{0}$. Therefore, $T_{0}=\min \{0, T\}$, i.e., $\Gamma_{t}^{\epsilon} \cap \Sigma_{t}=\emptyset$ for $t<\min \{0, T\}$.

To conclude, we note that, as $\Sigma_{t} \rightarrow \mathcal{C}(\Sigma)$ in $C_{\text {loc }}^{\infty}\left(\mathbb{R}^{n+1} \backslash\{\mathbf{0}\}\right)$ as $t \rightarrow 0$, we must have $\Gamma_{0}^{\epsilon} \cap \mathcal{C}(\Sigma) \subset\{\mathbf{0}\}$ by the previous discussion and parabolic maximum principle on bounded domains. However, if $\Gamma_{0}^{\epsilon} \cap \mathcal{C}(\Sigma)=\{\mathbf{0}\}$, then, as $\left\{\Gamma_{t}^{\epsilon}\right\}_{t \in[-1,0]}$ is smooth, its parabolic blowup at $O$ would be a static hyperplane and so $\Sigma$ would lie in a half-space whose boundary is this hyperplane. By [10, Lemma 3.25] and the fact that $\Sigma$ has polynomial volume growth, this could occur only if $\Sigma$ was the hyperplane - see also [5. Theorem 1.1]. This contradicts $\Sigma \in \mathcal{A C S}_{n}^{*}$ and shows Item (4). Finally, Item (5) follows from Proposition 3.2. Item (1) and Item (4) of Proposition 4.2

Following the arguments in [9], we show that, for elements of $\mathcal{A C S}_{n}^{*}$ with small entropy, the flow constructed in Proposition 4.4 exists for long time. A crucial ingredient in our proof is the fact that shrinker mean convexity is preserved under mean curvature flow i.e., Item (5) of Proposition 4.4

Proposition 4.5. If $\Sigma \in \mathcal{A C S}_{n}^{*}\left[\lambda_{n-1}\right]$ and, for $\epsilon \neq 0,\left\{\Gamma_{t}^{\epsilon}\right\}_{t \in[-1, T)}$ is given by Proposition 4.4 then $T=\infty$.

Proof. We argue by contradiction. If $T<\infty$, then Item (5) of Proposition 4.2, Item (2) of Proposition 4.4 and Brakke's local regularity theorem [3] or [26] imply that there is an $\mathbf{x}_{0} \in \bar{B}_{R(T)}$ so that the corresponding rescaled flow about $X_{0}=\left(\mathbf{x}_{0}, T\right)$,

$$
\tilde{\Gamma}_{s}^{\epsilon}=(T-t)^{-\frac{1}{2}}\left(\Gamma_{t}^{\epsilon}-\mathbf{x}_{0}\right), \quad s=-\log (T-t),
$$

satisfies that for some sequence $s_{i} \rightarrow \infty, \tilde{\Gamma}_{s_{i}}^{\epsilon}$ converges to a multiplicity one $F$-stationary varifold $\tilde{\Sigma}$ with $1<\lambda[\tilde{\Sigma}]<\lambda_{n-1}$ and

$$
\int_{\tilde{\Gamma}_{s_{i}}}\left|\mathbf{H}_{\tilde{\Gamma}_{s_{i}}}+\frac{\mathbf{x}^{\perp}}{2}\right|^{2} e^{-\frac{|\mathbf{x}|^{2}}{4}} d \mathcal{H}^{n} \rightarrow 0 .
$$

Invoking Items (3) and (4) of Proposition 4.2 and Item (1) of Proposition 4.4, it follows from Proposition 3.3 that on $\tilde{\Gamma}_{s_{i}}^{\epsilon} \cap B_{R_{i}}$ for $R_{i}=R(T) e^{s_{i} / 2}$,

$$
\left|A_{\tilde{\Gamma}_{s_{i}}}\right|<C\left(\Sigma, \Gamma^{\epsilon}, T\right)\left\{2\left(e^{-s_{i}}-T\right) H_{\tilde{\Gamma}_{s_{i}}}-e^{-\frac{s_{i}}{2}}\left(\mathbf{x}_{0}+e^{-\frac{s_{i}}{2}} \mathbf{x}\right) \cdot \mathbf{n}_{\tilde{\Gamma}_{s_{i}}}\right\} .
$$

Passing $s_{i} \rightarrow \infty$, this together with Brakke's local regularity theorem implies that

$$
\left|A_{\tilde{\Sigma}}\right| \leq-2 C T H_{\tilde{\Sigma}} \quad \text { on the regular set } \operatorname{Reg}(\tilde{\Sigma}) .
$$

If $n \geq 3$, then $\lambda_{n-1}<3 / 2$ and so $\tilde{\Sigma}$ is smoothly embedded by [9. Proposition 5.1]. If $n=2$, it follows from 4.56 and 4.57) that

$$
\int_{\tilde{\Gamma}_{s_{i}}^{\epsilon_{i}} \cap B_{R}}\left|A_{\tilde{\Gamma}_{s_{i}}^{\epsilon}}\right|^{2} e^{-\frac{|\mathbf{x}|^{2}}{4}} d \mathcal{H}^{2}<C^{\prime}\left(\lambda_{1}, R, T, C\right) .
$$


Thus, in view of [22, Lemma 4], the singular set $\operatorname{Sing}(\tilde{\Sigma})$ is discrete. Furthermore, as $\tilde{\Sigma}$ is of multiplicity one, it follows from Allard's regularity theorem that $\operatorname{Sing}(\tilde{\Sigma})=\emptyset$.

If $T=0$, then $\tilde{\Sigma}$ is flat and of multiplicity one and hence, $\lambda[\tilde{\Sigma}]=1$, contradicting $\lambda[\tilde{\Sigma}]>1$. If $T \neq 0$, then $H_{\tilde{\Sigma}}$ does not change sign and, as $F[\tilde{\Sigma}]<\lambda_{n-1}$, it follows from [10. Theorem 0.14] that $\tilde{\Sigma}$ is the self-shrinking sphere or a hyperplane. As $\tilde{\Gamma}_{s_{i}}^{\epsilon}$ is connected and non-compact, $\tilde{\Sigma}$ must be also non-compact and so $\tilde{\Sigma}$ cannot be a sphere. Hence, $\tilde{\Sigma}$ must be a hyperplane, yielding a contradiction as in the previous case.

We will need the following straightforward consequence of Propositions 4.4 and 4.5 in the next section.

Theorem 4.6. Let $\Sigma \in \mathcal{A C S}_{n}^{*}\left[\lambda_{n-1}\right]$ and let $\Gamma^{\epsilon}$ be as in Proposition 4.2 for $\epsilon \neq 0$. There is a unique smooth mean curvature flow $\left\{\Gamma_{t}^{\epsilon}\right\}_{t \in[-1,0]}$ with $\Gamma_{-1}^{\epsilon}=\Gamma^{\epsilon}$. Moreover, $\Gamma_{0}^{\epsilon}$ is a connected hypersurface which is smoothly asymptotic to, but disjoint from, $\mathcal{C}(\Sigma)$, and which satisfies $\mathbf{x} \cdot \mathbf{n}_{\Gamma_{0}^{\epsilon}}>0$.

\section{TOPOLOGY OF NON-COMPACT SHRINKERS OF SMALl ENTROPY}

We first observe the following topological fact which essentially says that the hypersurfaces $\Gamma_{0}^{\epsilon}$ from Theorem 4.6 are star-shaped relative to $\mathbf{0}$.

Proposition 5.1. Let $\Sigma \subset \mathbb{R}^{n+1}$ be a connected hypersurface which is smoothly asymptotic to a regular cone $\mathcal{C}(\Sigma)$. If $\mathbf{x} \cdot \mathbf{n}_{\Sigma}>0$ and $\Sigma \cap \mathcal{C}(\Sigma)=\emptyset$, then the map $\Pi: \Sigma \rightarrow \mathbb{S}^{n}$ given by

$$
\Pi(p)=\frac{\mathbf{x}(p)}{|\mathbf{x}(p)|}
$$

is a diffeomorphism onto its image. Furthermore, the image is a connected component of $\mathbb{S}^{n} \backslash \mathcal{L}(\Sigma)$, where $\mathcal{L}(\Sigma)$ is the link of $\mathcal{C}(\Sigma)$.

Proof. Let $\Omega=\Pi(\Sigma)$ and observe that, as $\Pi(\mathcal{C}(\Sigma))=\mathcal{L}(\Sigma)$ and $\Sigma \cap \mathcal{C}(\Sigma)=\emptyset, \Omega \cap$ $\mathcal{L}(\Sigma)=\emptyset$. It follows that $\Pi$ is a proper map. Indeed, suppose that $\mathcal{K} \subset \Omega$ is compact. As $\mathcal{K} \cap \mathcal{L}(\Sigma)=\emptyset$ and $\Sigma$ is smoothly asymptotic to $\mathcal{C}(\Sigma)$, there is an $R=R(\mathcal{K})>0$ so that $\Pi^{-1}(\mathcal{K}) \subset B_{R}$. As $\Sigma$ is a hypersurface, and so proper, $\Sigma \cap \bar{B}_{R}$ is compact and so $\Pi^{-1}(\mathcal{K}) \cap B_{R}$ is also compact.

Next notice that, as $\mathbf{x} \cdot \mathbf{n}_{\Sigma}>0$ and

$$
\mathrm{d} \Pi_{p}(\mathbf{v})=\frac{\mathbf{v}}{|\mathbf{x}(p)|}-\frac{\mathbf{x}(p)(\mathbf{x}(p) \cdot \mathbf{v})}{|\mathbf{x}(p)|^{3}} \text { for } \mathbf{v} \in T_{p} \Sigma,
$$

$\mathrm{d} \Pi \Pi_{p}$ is bijective for all $p \in \Sigma$. Hence, $\Pi$ is a local diffeomorphism and so $\Omega$ is an open subset of $\mathbb{S}^{n}$. As $\Omega$ is the image of a connected set, $\Omega$ itself must be connected and hence, by a standard topological result, $\Pi$ is a finite covering map.

We now show that $\Pi$ is a diffeomorphism. To see this, fix a $q \in \Omega$ and suppose that $p, p^{\prime} \in \Pi^{-1}(q)$ are two consecutive points in the pre-image of $q$ - here we order by distance from $\mathbf{0}$. For $\delta>0$ sufficiently small, let $\tilde{p}=\mathbf{x}(p)+\delta \mathbf{x}(q)$ and likewise define $\tilde{p}^{\prime}$. Invoking that $\mathbf{x} \cdot \mathbf{n}_{\Sigma}>0$, the straight line segment $\tilde{\tilde{p}} \tilde{p}^{\prime}$ connecting $\tilde{p}$ to $\tilde{p}^{\prime}$ intersects transversally with $\Sigma$ exactly once. Now let $\Pi_{\Sigma}$ be the nearest point projection onto $\Sigma$ and let $\gamma:[0,1] \rightarrow \Sigma$ be a simple, smooth parametrized curve connecting $\Pi_{\Sigma}(\tilde{p})$ to $\Pi_{\Sigma}\left(\tilde{p}^{\prime}\right)$. Consider the curve $\tilde{\gamma}:[0,1] \rightarrow \mathbb{R}^{n+1}$ defined by

$$
\tilde{\gamma}(t)=\mathbf{x}(\gamma(t))+\delta \mathbf{n}_{\Sigma}(\gamma(t))
$$




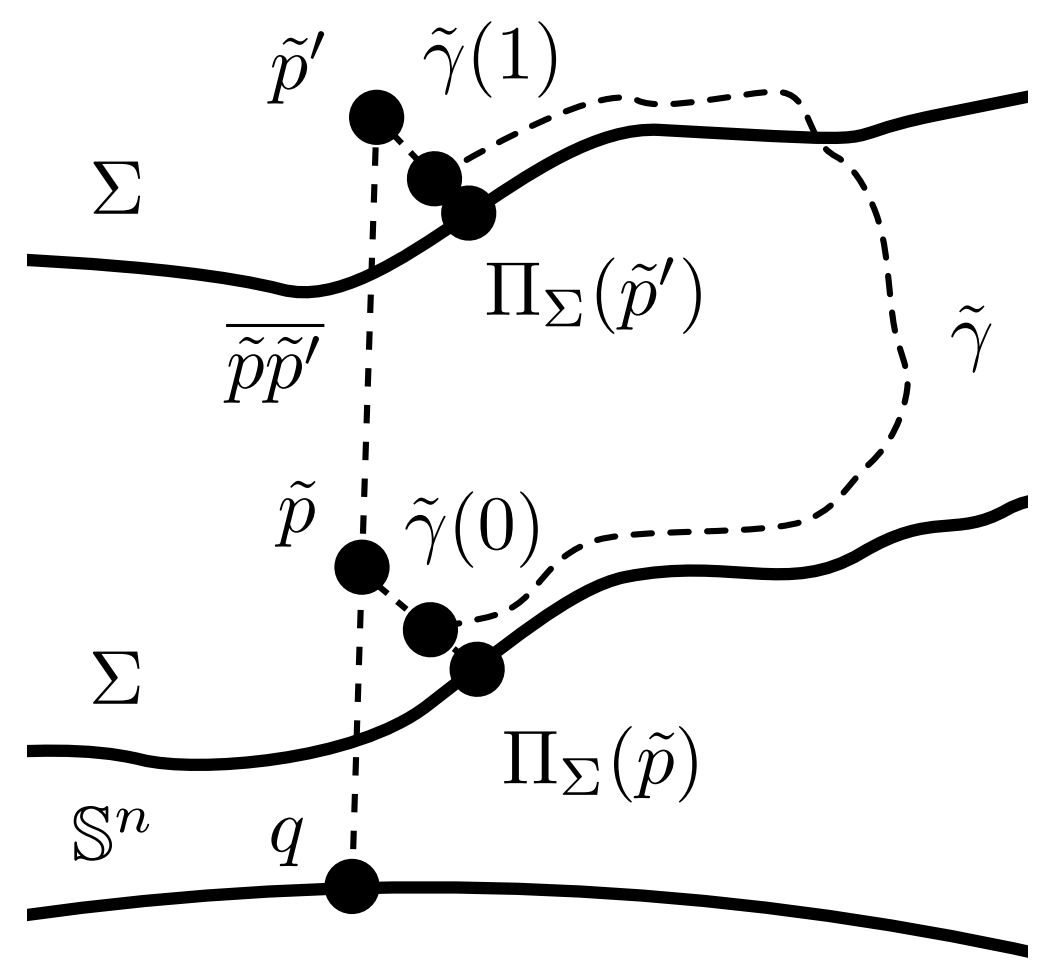

FIGURE 1. The line segment $\overline{\tilde{p}} \tilde{p}^{\prime}$ and curve $\tilde{\gamma}$.

Clearly, $\overline{\tilde{p} \tilde{\gamma}(0)} \cup \tilde{\gamma} \cup \overline{\tilde{\gamma}(1) \tilde{p}^{\prime}}$ is homotopic to $\overline{\tilde{p} \tilde{p}^{\prime}}$ in $\mathbb{R}^{n+1}$, but does not intersect $\Sigma$. This is a contradiction and completes the proof that $\Pi$ is a diffeomorphism of $\Sigma$ onto $\Omega$.

Finally, we show that $\Omega$ must be a connected component of $\mathbb{S}^{n} \backslash \mathcal{L}(\Sigma)$. To see this, let $\hat{\Omega}$ be the connected component of $\mathbb{R}^{n+1} \backslash \mathcal{C}(\Sigma)$ which contains $\Sigma$ - such a component exists as $\Sigma$ is connected and disjoint from $\mathcal{C}(\Sigma)$. Clearly, $\Omega \subset \hat{\Omega} \cap \mathbb{S}^{n}$ and $\hat{\Omega} \cap \mathbb{S}^{n}$ is a connected component of $\mathbb{S}^{n} \backslash \mathcal{L}(\Sigma)$. Pick a point $q \in \partial \Omega$ and a sequence $q_{i} \in \Omega$ with $q_{i} \rightarrow q \notin \Omega$. Set $p_{i}=\Pi^{-1}\left(q_{i}\right) \in \Sigma$. As $\Sigma$ is proper, $\left|\mathbf{x}\left(p_{i}\right)\right| \rightarrow \infty$ and so, as $\Sigma$ is smoothly asymptotic to $\mathcal{C}(\Sigma), q \in \mathcal{L}(\Sigma)$. Hence, $\partial \Omega \subset \mathcal{L}(\Sigma)$ and our claim is verified.

We may now prove Theorem 1.1

Proof of Theorem 1.1. First observe that the theorem is trivially true for hyperplanes. We next claim that $\Sigma$ must be connected. Indeed, otherwise one could use two of the connected components as barriers to find a stable self-shrinker which is impossible in view of [1. Lemma 3.3]. As such, we may assume that $\Sigma \in \mathcal{A C S}_{n}^{*}\left[\lambda_{n-1}\right]$.

Hence, applying Theorem 4.6, we associate to $\Sigma$ two smooth mean curvature flows $\left\{\Gamma_{t}^{ \pm \epsilon}\right\}_{t \in[-1,0]}$ with $\Gamma_{-1}^{ \pm \epsilon}=\Gamma^{ \pm \epsilon}$ for some $\epsilon>0$ small, where $\Gamma^{ \pm \epsilon}$ are the hypersurfaces as in Proposition 4.2 Moreover, each $\Gamma^{ \pm}=\Gamma_{0}^{ \pm \epsilon}$ is a connected star-shaped hypersurface which is smoothly asymptotic to, but disjoint from, $\mathcal{C}(\Sigma)$. Notice that $\mathbb{R}^{n+1} \backslash \Sigma$ consists of exactly two connected components (as $\Sigma$ is connected) one containing $\Gamma^{-\epsilon}$ and the other $\Gamma^{\epsilon}$. Hence, $\mathbb{R}^{n+1} \backslash \mathcal{C}(\Sigma)$ also consists of exactly two connected components $\hat{\Omega}^{ \pm}$each 
containing $\Gamma^{ \pm}$and so, by Proposition 5.1. each $\Gamma^{ \pm}$is diffeomorphic to $\Omega^{ \pm}=\hat{\Omega}^{ \pm} \cap \mathbb{S}^{n}$. This proves all but the last claim.

The final claim, that $\mathcal{L}(\Sigma)$ is connected, follows by applying the Mayer-Vietoris long exact sequence for reduced homology. Indeed, slightly enlarge $\Omega^{ \pm}$so that both are still connected and $\Omega^{+} \cap \Omega^{-}$is a regular tubular neighborhood of $\mathcal{L}(\Sigma)$ in $\mathbb{S}^{n}$. One then has

$$
\tilde{H}_{1}\left(\mathbb{S}^{n}\right) \rightarrow \tilde{H}_{0}(\mathcal{L}(\Sigma)) \rightarrow \tilde{H}_{0}\left(\Omega^{-}\right) \oplus \tilde{H}_{0}\left(\Omega^{+}\right)
$$

as part of the long exact sequence. As $n \geq 2$ and both $\Omega^{-}$and $\Omega^{+}$are connected,

$$
\{0\}=\tilde{H}_{1}\left(\mathbb{S}^{n}\right)=\tilde{H}_{0}\left(\Omega^{-}\right)=\tilde{H}_{0}\left(\Omega^{+}\right) .
$$

Hence, $\tilde{H}_{0}(\mathcal{L}(\Sigma))=\{0\}$ and so $\mathcal{L}(\Sigma)$ is connected.

We next prove Corollary 1.2 .

Proof of Corollary 1.2 First, notice that all possible singularities at the first singular time of mean curvature flow of closed surfaces have smooth supports - see [17, Theorem 2]. Hence, one can strengthen [9. Theorem 0.8] of Colding-Ilmanen-Minicozzi-White to conclude that any closed self-shrinker with entropy (equivalently, Gaussian surface area) less than or equal to $\lambda_{1}$ is diffeomorphic to a sphere. Furthermore, such a self-shrinker must be $\mathbb{S}_{*}^{2}$ by a recent result of Brendle [4] on the uniqueness of closed genus zero self-shrinkers (recall, in our definition self-shrinkers are automatically embedded).

Next, as observed in [2, Proposition 4.7] and in the proof of [2, Theorem 1.2] when $n=2$, any non-compact self-shrinker in $\mathbb{R}^{3}$ with $\lambda[\Sigma] \leq \lambda_{1}$ is either a rotation of $\mathbb{S}_{*}^{1} \times \mathbb{R}$ or asymptotically conical. By Theorem 1.1 , in the latter case, $\Sigma$ is diffeomorphic to a open disk. Invoking [4. Theorem 2] of Brendle, it follows that $\Sigma$ must be flat. Thus, the only self-shrinkers with entropy less than or equal to $\lambda_{1}$ are $\mathbb{S}_{*}^{2}$ and rotations of $\mathbb{R}^{2} \times\{0\}$ and $\mathbb{S}_{*}^{1} \times \mathbb{R}$

To prove the gap, we argue by contradiction. Namely, suppose that there was no such $\delta_{0}$. Then there would exist a sequence $\Sigma_{i}$ of self-shrinkers with $\lambda\left[\Sigma_{i}\right] \in\left(\lambda_{1}, 2\right)$ and $\lambda\left[\Sigma_{i}\right] \rightarrow \lambda_{1}$. Up to passing to a subsequence, we may assume that $\Sigma_{i}$ converges to a multiplicity one $F$-stationary varifold $\Sigma$ with $\lambda[\Sigma] \leq \lambda_{1}$. As each $\Sigma_{i}$ is two-sided, the tangent cones of $\Sigma$ cannot be quasi-planes. Also, there is no minimal cone with isolated singularities in $\mathbb{R}^{3}$. Thus, by Allard's regularity theorem, $\Sigma_{i}$ converges in $C_{\text {loc }}^{\infty}\left(\mathbb{R}^{3}\right)$ to $\Sigma$ and $\lambda[\Sigma]=\lim _{i \rightarrow \infty} \lambda\left[\Sigma_{i}\right]=\lambda_{1}$. By the rigidity of self-shrinkers of small entropy established in the previous paragraph, this implies that (up to a rotation) $\Sigma=\mathbb{S}_{*}^{1} \times \mathbb{R}$. Hence, in view of [8, Theorem 0.1], for all $i$ sufficiently large, $\Sigma_{i}$ are rotations of $\mathbb{S}_{*}^{1} \times \mathbb{R}$ and, in particular, $\lambda\left[\Sigma_{i}\right]=\lambda_{1}$, giving the desired contradiction.

Finally we show Corollary 1.3 .

Proof of Corollary 1.3. Let $\left\{\Sigma_{t}\right\}_{t \in[-1, T)}$ be the maximal smooth mean curvature flow with initial surface $\Sigma_{-1}=\Sigma$. As $\Sigma$ is closed, a comparison with a large shrinking sphere implies that $T<\infty$. Let $X_{0}=\left(\mathrm{x}_{0}, T\right)$ be a singular point of the flow. It follows from Corollary 1.2 and the monotonicity of entropy that $\lambda[\Sigma] \geq \lambda_{2}$ with equality if and only if, modulo translations and scalings, $\Sigma$ is equal to $\mathbb{S}^{2}$. If the tangent flow at $X_{0}$ is a selfshrinking sphere, then, by Brakke's local regularity theorem, $\Sigma_{t}$ must be very close to the sphere for all $t$ near $T$. In particular, $\Sigma_{t}$ is diffeomorphic to $\mathbb{S}^{2}$ and thus, as the flow is smooth, so is $\Sigma$. Hence, if $\Sigma$ has positive genus, the tangent flow cannot be a shrinking sphere and so $\lambda[\Sigma]>\lambda_{1}$ by the monotonicity formula and Corollary 1.2 . 


\section{APPENDIX A.}

We use a weighted version of Huisken's monotonicity formula to establish a parabolic maximum principle for smooth non-compact mean curvature flows with boundaries. This is a slight generalization of [12, Corollary 1.1].

Theorem A.1. Let $\mathcal{S}=\left\{\Sigma_{t}\right\}_{t \in[-1, T)}$ be a smooth mean curvature flow in $\mathbb{R}^{n+1}$ with finite entropy. For a fixed $R \geq 0$ and $X_{0}=(\mathbf{0},-1)$, suppose that $u$ is a $C^{2}$ function on $\mathcal{S} \backslash C_{R, T+1}\left(X_{0}\right)$ which satisfies:

(1) on $\stackrel{\mathcal{S}}{=} \mathcal{S} \backslash \overline{C_{R, T+1}\left(X_{0}\right)}$,

$$
\left(\frac{d}{d t}-\Delta_{\Sigma_{t}}\right) u \geq \mathbf{a} \cdot \nabla_{\Sigma_{t}} u+b u
$$

with $\sup _{\mathcal{S}}|\mathbf{a}|^{2}+|b|=M_{0}<\infty$;

(2) $u>c_{0} \geq 0$ on $\partial_{P} \mathcal{S}=\left(\Sigma_{-1} \backslash B_{R}\right) \cup\left(\mathcal{S} \cap \partial C_{R, T+1}\left(X_{0}\right)\right)$ for some constant $c_{0}$;

(3) for all $t \in[-1, T)$,

$$
\int_{\Sigma_{t} \backslash B_{R}}\left(|u|^{2}+\left|\frac{d u}{d t}\right|^{2}+\left|\nabla_{\Sigma_{t}} u\right|^{2}+\left|\nabla_{\Sigma_{t}}^{2} u\right|^{2}\right) \Phi_{(\mathbf{0}, T)} d \mathcal{H}^{n}<\infty
$$

where

$$
\Phi_{(\mathbf{0}, T)}(\mathbf{x}, t)=(T-t)^{-\frac{n}{2}} \Phi\left(\frac{\mathbf{x}}{\sqrt{T-t}}\right)=(4 \pi(T-t))^{-\frac{n}{2}} e^{-\frac{|\mathbf{x}|^{2}}{4(T-t)}} .
$$

Then for all $t \in[-1, T)$,

$$
\inf _{\Sigma_{t} \backslash B_{R}} u \geq c_{0} e^{M_{1}(t+1)} \geq 0
$$

where $M_{1}=\min \left\{\inf _{\mathcal{S}} b, 0\right\} \leq 0$.

Proof. We will first show that $u \geq 0$. Let $u_{0}(p, t)=\max \{-u(p, t), 0\} \geq 0$ on $\mathcal{\mathcal { S }}$ and we extend $u_{0}$ by zero to the whole $\mathcal{S}$. As $u$ is continuous and, for every $0<T^{\prime}<$ $T, \mathcal{S} \cap \partial_{P} C_{R, T^{\prime}+1}\left(X_{0}\right)$ is compact, Item (2) implies that $-u$ is strictly negative in a neighborhood of $\mathcal{S} \cap \partial_{P} C_{R, T^{\prime}+1}\left(X_{0}\right)$. Hence, $u_{0}^{2}$ is $C^{1,1}$ and satisfies, in the weak sense, that

$$
\left(\frac{d}{d t}-\Delta_{\Sigma_{t}}\right) u_{0}^{2} \leq-2\left|\nabla_{\Sigma_{t}} u_{0}\right|^{2}+2 u_{0} \mathbf{a} \cdot \nabla_{\Sigma_{t}} u_{0}+2 b u_{0}^{2} \leq 3 M_{0} u_{0}^{2} .
$$

Here we used Item (1) and Young's inequality.

Invoking (A.5) and Item (3), it follows from the weighted monotonicity formula [11, Theorem 4.13] that

$$
\frac{d}{d t} \int_{\Sigma_{t}} u_{0}^{2} \Phi_{(\mathbf{0}, T)} d \mathcal{H}^{n} \leq 3 M_{0} \int_{\Sigma_{t}} u_{0}^{2} \Phi_{(\mathbf{0}, T)} d \mathcal{H}^{n} .
$$

This together with Item (2) implies that for all $t \in[-1, T)$,

$$
\int_{\Sigma_{t}} u_{0}^{2} \Phi_{(\mathbf{0}, T)} d \mathcal{H}^{n}=0
$$

That is, $u_{0} \equiv 0$ on $\mathcal{S}$ and so $u \geq 0$ as claimed.

Set $\tilde{u}=e^{-M_{1}(t+1)} u$. Using $\tilde{u} \geq 0$ and $b-M_{1} \geq 0$, it follows that

$$
\left(\frac{d}{d t}-\Delta_{\Sigma_{t}}\right) \tilde{u} \geq \mathbf{a} \cdot \nabla_{\Sigma_{t}} \tilde{u}+\left(b-M_{1}\right) \tilde{u} \geq \mathbf{a} \cdot \nabla_{\Sigma_{t}} \tilde{u} .
$$


As $M_{1} \leq 0, \tilde{u}>c_{0}$ on $\partial_{P} \mathcal{S}$. Applying the same argument as above to $\tilde{u}-c_{0}$, we conclude that $\tilde{u} \geq c_{0}$ which completes the proof.

\section{APPENDIX B.}

Throughout this appendix, let $\Sigma$ be a self-shrinker in $\mathbb{R}^{n+1}$ and let $H_{w}^{1}(\Sigma)$ and $L_{w}^{2}(\Sigma)$ be as in the proof of Proposition 4.1. The goal is to prove the natural embedding

$$
\iota: H_{w}^{1}(\Sigma) \hookrightarrow L_{w}^{2}(\Sigma)
$$

is compact.

First, we prove a lemma that yields a uniform decay rate for the weighted $L^{2}$ integration over exterior regions.

Lemma B.1. For all $\phi \in C_{c}^{\infty}(\Sigma)$,

$$
\int_{\Sigma} \phi^{2}|\mathbf{x}|^{2} e^{-\frac{|\mathbf{x}|^{2}}{4}} d \mathcal{H}^{n} \leq 16 \int_{\Sigma}\left|\nabla_{\Sigma} \phi\right|^{2} e^{-\frac{|\mathbf{x}|^{2}}{4}} d \mathcal{H}^{n}+4 n \int_{\Sigma} \phi^{2} e^{-\frac{|\mathbf{x}|^{2}}{4}} d \mathcal{H}^{n}
$$

Proof. Recall that the divergence theorem implies that

$$
\int_{\Sigma} \operatorname{div}_{\Sigma} \mathbf{V} d \mathcal{H}^{n}=-\int_{\Sigma} \mathbf{H}_{\Sigma} \cdot \mathbf{V} d \mathcal{H}^{n}
$$

for all vector fields $\mathbf{V}$ of compact support. Setting $\mathbf{V}=\mathbf{x} \phi^{2} e^{-|\mathbf{x}|^{2} / 4}$ yields the lemma.

Indeed, we compute

$$
\operatorname{div}_{\Sigma} \mathbf{V}=n \phi^{2} e^{-\frac{|\mathbf{x}|^{2}}{4}}+2\left(\mathbf{x} \cdot \nabla_{\Sigma} \phi\right) \phi e^{-\frac{|\mathbf{x}|^{2}}{4}}-\frac{1}{2} \phi^{2}\left|\mathbf{x}^{\top}\right|^{2} e^{-\frac{|\mathbf{x}|^{2}}{4}}
$$

and, using the self-shrinker equation and Young's inequality,

$$
\begin{aligned}
\frac{1}{2} \int_{\Sigma} \phi^{2}|\mathbf{x}|^{2} e^{-\frac{|\mathbf{x}|^{2}}{4}} d \mathcal{H}^{n} & =n \int_{\Sigma} \phi^{2} e^{-\frac{|\mathbf{x}|^{2}}{4}} d \mathcal{H}^{n}+2 \int_{\Sigma}\left(\mathbf{x} \cdot \nabla_{\Sigma} \phi\right) \phi e^{-\frac{|\mathbf{x}|^{2}}{4}} d \mathcal{H}^{n} \\
& \leq n \int_{\Sigma} \phi^{2} e^{-\frac{|\mathbf{x}|^{2}}{4}} d \mathcal{H}^{n}+4 \int_{\Sigma}\left|\nabla_{\Sigma} \phi\right|^{2} e^{-\frac{|\mathbf{x}|^{2}}{4}} d \mathcal{H}^{n} \\
& +\frac{1}{4} \int_{\Sigma} \phi^{2}|\mathbf{x}|^{2} e^{-\frac{|\mathbf{x}|^{2}}{4}} d \mathcal{H}^{n}
\end{aligned}
$$

The desired estimate follows from a rearrangement of the above inequality.

Now we are ready to prove that

Proposition B.2. The natural embedding $\iota: H_{w}^{1}(\Sigma) \hookrightarrow L_{w}^{2}(\Sigma)$ is compact.

Proof. As $C_{c}^{\infty}(\Sigma)$ is dense in $H_{w}^{1}(\Sigma)$, it suffices to prove the proposition for $\iota$ restricted to $C_{c}^{\infty}(\Sigma) \cap H_{w}^{1}(\Sigma)$. Namely, let $\phi_{i}$ be a sequence of elements of $C_{c}^{\infty}(\Sigma) \cap H_{w}^{1}(\Sigma)$ with $\left(\phi_{i}, \phi_{i}\right)_{1} \leq C$ for some constant $C>0$. We need to find a subsequence $\phi_{i_{j}}$ converging strongly in $L_{w}^{2}(\Sigma)$.

For $R>1$ we introduce the cut-off function $\psi_{R}: \mathbb{R}^{n+1} \rightarrow[0,1]$ so that $\psi_{R}=1$ in $B_{R}$, $\psi_{R}=0$ outside $B_{2 R}$, and $\left|D \psi_{R}\right| \leq 2 / R$. Let $\phi_{i}^{R}=\phi_{i} \psi_{R}$. Clearly $\operatorname{spt}\left(\phi_{i}^{R}\right) \subset \Sigma \cap B_{2 R}$ and $\left(\phi_{i}^{R}, \phi_{i}^{R}\right)_{1} \leq 8 C$ for all $i$. By the Rellich-Kondrachov compactness theorem, there is a subsequence of $\phi_{i}^{R}$ converging strongly in $L_{w}^{2}(\Sigma)$ to $\phi^{R}$ supported in $\Sigma \cap B_{2 R}$. Now take an increasing sequence $R_{k} \rightarrow \infty$ and by a diagonalization argument we find a subsequence $i_{j} \rightarrow \infty$ and $\phi \in L_{w}^{2}(\Sigma)$ so that $\phi_{i_{j}} \rightarrow \phi$ strongly in $L_{w}^{2}\left(\Sigma \cap B_{R_{k}}\right)$ for each $k$. 
Observe that $\left\|\phi^{R_{k}}-\phi\right\|_{0} \rightarrow 0$ as $k \rightarrow \infty$. And by Lemma B.1, $\left\|\phi_{i_{j}}-\phi_{i_{j}}^{R_{k}}\right\|_{0} \leq C^{\prime} R_{k}^{-2}$ for some $C^{\prime}$ depending only on $n$ and $C$. Thus

$$
\left\|\phi_{i_{j}}-\phi\right\|_{0} \leq\left\|\phi_{i_{j}}-\phi_{i_{j}}^{R_{k}}\right\|_{0}+\left\|\phi_{i_{j}}^{R_{k}}-\phi^{R_{k}}\right\|_{0}+\left\|\phi^{R_{k}}-\phi\right\|_{0} \rightarrow 0
$$

i.e., $\phi_{i_{j}} \rightarrow \phi$ strongly in $L_{w}^{2}(\Sigma)$.

\section{APPENDIX C.}

Let $\Sigma \in \mathcal{A C S}_{n}^{*}$ and let $f$ be the lowest eigenfunction of $L_{\Sigma}$ as in Proposition 4.1. Throughout $\epsilon_{1}>0$ is assumed to be sufficiently small. We consider a one-parameter family of hypersurfaces

$$
\Gamma^{s}=\left\{\mathbf{y} \in \mathbb{R}^{n+1}: \mathbf{y}=\mathbf{x}(p)+s f(p) \mathbf{n}_{\Sigma}(p), p \in \Sigma\right\}
$$

for $|s| \leq \epsilon_{1}$ with $\Gamma^{0}=\Sigma$. Observe that, as $\Gamma^{s}$ is asymptotically conical by Item (3) of Proposition 4.2 for each $|s| \leq \epsilon_{1}$, this family of hypersurfaces is a normal variation of $\Gamma^{s}$ with vector field $f_{\Gamma^{s}} \mathbf{n}_{\Gamma^{s}}$, where $f_{\Gamma^{s}}=f \mathbf{n}_{\Sigma} \cdot \mathbf{n}_{\Gamma^{s}}$. Define a function

(C.2) $G: \mathbb{R}^{n+1} \times \mathbb{R}^{+} \times\left[-\epsilon_{1}, \epsilon_{1}\right] \rightarrow \mathbb{R}^{+}, \quad G\left(\mathbf{x}_{0}, t_{0}, s\right)=\left(4 \pi t_{0}\right)^{-\frac{n}{2}} \int_{\Gamma^{s}} e^{-\frac{\left|\mathbf{x}-\mathbf{x}_{0}\right|^{2}}{4 t_{0}}} d \mathcal{H}^{n}$.

The goal of this appendix is to show that there is an $\epsilon_{2} \in\left(0, \epsilon_{1}\right)$ sufficiently small so that if $s \neq 0$ and $|s| \leq \epsilon_{2}$, then

$$
\lambda\left[\Gamma^{s}\right] \equiv \sup _{\mathbf{x}_{0}, t_{0}} G\left(\mathbf{x}_{0}, t_{0}, s\right)<G(\mathbf{0}, 1,0)=\lambda[\Sigma] .
$$

In order to establish (C.3, we follow closely the proof of [10, Theorem 0.15] and thus it suffices to show:

\section{Proposition C.1. The following properties hold:}

(1) $G$ has a strict local maximum at $(\mathbf{0}, 1,0)$;

(2) The restriction of $G$ to $\Sigma$, i.e., $G\left(\mathbf{x}_{0}, t_{0}, 0\right)$, has a strict global maximum at $(\mathbf{0}, 1)$;

(3) $\left|\partial_{s} G\right|$ is uniformly bounded on compact sets;

(4) $G\left(\mathbf{x}_{0}, t_{0}, s\right)$ is strictly less than $G(\mathbf{0}, 1,0)$ whenever $\left|\mathbf{x}_{0}\right|$ is sufficiently large;

(5) $G\left(\mathbf{x}_{0}, t_{0}, s\right)$ is strictly less than $G(\mathbf{0}, 1,0)$ whenever $\left|\log t_{0}\right|$ is sufficiently large.

Proof. Observe that, by [10, Proposition 3.6], the gradient of $G$ vanishes at $(\mathbf{0}, 1,0)$. Hence, Item (1) will follow if we show that the Hessian of $G$ at $(\mathbf{0}, 1,0)$ is negative definite. That is, if, for any $a, b \in \mathbb{R}$ and $\mathbf{y} \in \mathbb{R}^{n+1}$ so that $(a, b, \mathbf{y}) \neq(0,0, \mathbf{0})$,

$$
\left.\partial_{s s}\right|_{s=0} G(s \mathbf{y}, 1+a s, b s)<0 .
$$

To see this, we first use [10, Theorem 4.14] to conclude that, at $s=0$,

$$
\begin{aligned}
\partial_{s s} G(s \mathbf{y}, 1+a s, s)=(4 \pi)^{-\frac{n}{2}} & \int_{\Sigma}\left(-f L_{\Sigma} f-a^{2} H_{\Sigma}^{2}-\frac{\left(\mathbf{y} \cdot \mathbf{n}_{\Sigma}\right)^{2}}{2}\right) e^{-\frac{|\mathbf{x}|^{2}}{4}} d \mathcal{H}^{n} \\
& +(4 \pi)^{-\frac{n}{2}} \int_{\Sigma}\left(2 a f H_{\Sigma}+f \mathbf{y} \cdot \mathbf{n}_{\Sigma}\right) e^{-\frac{|\mathbf{x}|^{2}}{4}} d \mathcal{H}^{n}
\end{aligned}
$$

Note that $f, H_{\Sigma}$, and $\mathbf{y} \cdot \mathbf{n}_{\Sigma}$ are eigenfunctions corresponding to different eigenvalues of $L_{\Sigma}$. As $\Sigma$ is asymptotically conical and $f$ satisfies 4.2 , and (4.3), it follows from [10, 
Corollary 3.10] that $f$ is orthogonal to both $H_{\Sigma}$ and $\mathbf{y} \cdot \mathbf{n}_{\Sigma}$ in $L_{w}^{2}(\Sigma)$. This implies that the last integration in the right hand side of C.5 is zero and so, at $s=0$,

$$
\begin{aligned}
\partial_{s s} G(s \mathbf{y}, 1+a s, s) & =(4 \pi)^{-\frac{n}{2}} \int_{\Sigma}\left(-f L_{\Sigma} f-a^{2} H_{\Sigma}^{2}-\frac{\left(\mathbf{y} \cdot \mathbf{n}_{\Sigma}\right)^{2}}{2}\right) e^{-\frac{|\mathbf{x}|^{2}}{4}} d \mathcal{H}^{n} \\
& \leq \mu(4 \pi)^{-\frac{n}{2}} \int_{\Sigma} f^{2} e^{-\frac{|\mathbf{x}|^{2}}{4}} d \mathcal{H}^{n}<0 .
\end{aligned}
$$

Hence, for any $b \in \mathbb{R} \backslash\{0\}$, the second derivative $\left.\partial_{s s}\right|_{s=0} G(s \mathbf{y}, 1+s a, b s)<0$. Finally, appealing to [10, Theorem 4.14] again,

(C.7) $\left.\partial_{s s}\right|_{s=0} G(s \mathbf{y}, 1+s a, 0)=(4 \pi)^{-\frac{n}{2}} \int_{\Sigma}\left(-a^{2} H_{\Sigma}^{2}-\frac{\left(\mathbf{y} \cdot \mathbf{n}_{\Sigma}\right)^{2}}{2}\right) e^{-\frac{|\mathbf{x}|^{2}}{4}} d \mathcal{H}^{n}<0$,

where the inequality follows from the fact that $\Sigma \in \mathcal{A C S}_{n}^{*}$ and so $\Sigma$ can neither split off a line isometrically (and so is non-flat) nor can $H_{\Sigma}$ cannot vanish identically. Hence, we conclude that the Hessian of $G$ at $(\mathbf{0}, 1,0)$ is negative definite as claimed. In particular, there is an $\epsilon_{3} \in\left(0, \epsilon_{1}\right)$ so that

(C.8) $\quad G\left(\mathbf{x}_{0}, t_{0}, s\right)<G(\mathbf{0}, 1,0)$ if $0<\left|\mathbf{x}_{0}\right|^{2}+\left(\log t_{0}\right)^{2}+s^{2}<\epsilon_{3}^{2}$.

Next, [10, Lemma 7.10] implies that $\lambda[\Sigma]=G(\mathbf{0}, 1,0)$, and there is an $\alpha_{0}>0$ so that

$$
G\left(\mathbf{x}_{0}, t_{0}, 0\right)<G(\mathbf{0}, 1,0)-\alpha_{0} \quad \text { if }\left|\mathbf{x}_{0}\right|^{2}+\left(\log t_{0}\right)^{2}>\frac{\epsilon_{3}^{2}}{4}
$$

which gives Item (2).

As $\lambda[\Sigma]<\infty, \Sigma$ has bounded area ratios. Since $\Sigma$ has uniformly bounded second fundamental form, combining with 4.2 , and (4.3), it follows from [25, (2.34)] that

$$
\sup _{|s| \leq \epsilon_{1}} \sup _{\mathbf{x} \in \mathbb{R}^{n+1}, r>1} r^{-n} \mathcal{H}^{n}\left(\Gamma^{s} \cap B_{r}(\mathbf{x})\right)<C\left(\Sigma, C_{0}, C_{1}\right)
$$

Furthermore, as $\Sigma$ is asymptotically conical, by (4.2) and 4.3), there is a $C_{H}$ depending only on $C_{0}, C_{1}, C_{2}$, and $\Sigma$ so that

$$
\sup _{|s| \leq \epsilon_{1}} \sup _{\Gamma^{s}} H_{\Gamma^{s}}^{2} \leq C_{H}
$$

Then, using the first variation formula [10, Lemma 3.1], we argue as in [10, (7.5)] to get that

$$
\partial_{t_{0}} G\left(\mathbf{x}_{0}, t_{0}, s\right) \geq-\left(4 \pi t_{0}\right)^{-\frac{n}{2}} \int_{\Gamma^{s}} \frac{H_{\Gamma^{s}}^{2}}{4} e^{-\frac{\left|\mathbf{x}-\mathbf{x}_{0}\right|^{2}}{4 t_{0}}} d \mathcal{H}^{n} \geq-\frac{C_{H}}{4} G\left(\mathbf{x}_{0}, t_{0}, s\right) .
$$

Thus, for $0<t_{0} \leq \bar{t}$,

$$
G\left(\mathbf{x}_{0}, t_{0}, s\right) \leq e^{\frac{C_{H} \bar{t}}{4}} G\left(\mathbf{x}_{0}, \bar{t}, s\right)
$$

In particular, choosing $\bar{t}=1$, this together with C.10 implies that

$$
\sup _{|s| \leq \epsilon_{1}} \sup _{\mathbf{x} \in \mathbb{R}^{n+1}, r>0} r^{-n} \mathcal{H}^{n}\left(\Gamma^{s} \cap B_{r}(\mathbf{x})\right)<C_{M},
$$

where $C_{M}$ depends only on $n, C$, and $C_{H}$.

An easy consequence of C.14 is that given $\alpha_{1}>0$ small, there is an $R>0$ so that

$$
\sup _{|s| \leq \epsilon_{1}} \sup _{\mathbf{x}_{0}, t_{0}}\left(4 \pi t_{0}\right)^{-\frac{n}{2}} \int_{\Gamma^{s} \backslash B_{R \sqrt{t_{0}}}\left(\mathbf{x}_{0}\right)} \frac{\left|\mathbf{x}-\mathbf{x}_{0}\right|}{\sqrt{t_{0}}} e^{-\frac{\left|\mathbf{x}-\mathbf{x}_{0}\right|^{2}}{4 t_{0}}} d \mathcal{H}^{n}<\frac{\alpha_{1}}{4} \text {. }
$$


Invoking the first variation formula again, we get that

$$
\partial_{s} G\left(\mathbf{x}_{0}, t_{0}, s\right)=\left(4 \pi t_{0}\right)^{-\frac{n}{2}} \int_{\Gamma^{s}} f_{\Gamma^{s}}\left(H_{\Gamma^{s}}-\frac{\left(\mathbf{x}-\mathbf{x}_{0}\right) \cdot \mathbf{n}_{\Gamma^{s}}}{2 t_{0}}\right) e^{-\frac{\left|\mathbf{x}-\mathbf{x}_{0}\right|^{2}}{4 t_{0}}} d \mathcal{H}^{n} .
$$

Let $\mathcal{K}$ be any compact set in $\mathbb{R}^{n+1} \times \mathbb{R}^{+} \times\left[-\epsilon_{1}, \epsilon_{1}\right]$. There is an $\bar{R}$ so that $B_{\bar{R}}$ contains $B_{R \sqrt{t_{0}}}\left(\mathbf{x}_{0}\right)$ for every $\left(\mathbf{x}_{0}, t_{0}, s\right) \in \mathcal{K}$. Thus, with 4.2) and (C.11), it follows from (C.15) that the integration in $\left(\mathrm{C} .16\right.$ restricted on $\mathbb{R}^{n+1} \backslash B_{\bar{R}}$ will be uniformly bounded on $\mathcal{K}$. Clearly, the integration in (C.16) restricted on $B_{2 \bar{R}}$ is continuous in all three variables $\mathbf{x}_{0}$, $t_{0}$, and $s$. Hence we conclude that $\partial_{s} G$ is uniformly bounded on $\mathcal{K}$, proving Item (3).

To see Item (4), observe that, by Item (3) of Proposition 4.2 for any given $\alpha_{1}>0$ small, there is an $R^{\prime}>1$ so that if $\left|\mathbf{x}_{0}\right|>R^{\prime}, 0<t_{0} \leq 1$, and $|s| \leq \epsilon_{1}$, then either $\left(\Gamma^{s}-\mathbf{x}_{0}\right) / \sqrt{t_{0}}$ is contained outside $B_{R}$, or $\left(\Gamma^{s}-\mathbf{x}_{0}\right) / \sqrt{t_{0}}$ in $B_{2 R}$ is a connected small $C^{1}$ graph over some hyperplane. This, together with (C.15), implies that $G\left(\mathbf{x}_{0}, t_{0}, s\right)<1+\alpha_{1} / 2$.

Next we study the region where $t_{0}>1$ and $\left|\mathbf{x}_{0}\right|$ sufficiently large. Set $\rho=1 / \sqrt{t_{0}}<1$ and $\mathbf{y}_{0}=\mathbf{x}_{0} / \sqrt{t_{0}}$. By [C.14], given $\alpha_{1}>0$ small, there is an $r_{1}>0$ small so that for all $\mathbf{y}_{0} \in \mathbb{R}^{n+1}, \rho<1$, and $|s| \leq \epsilon_{1}$,

$$
(4 \pi)^{-\frac{n}{2}} \int_{\rho \Gamma^{s} \cap B_{r_{1}}} e^{-\frac{\left|\mathbf{x}-\mathbf{y}_{0}\right|^{2}}{4}} d \mathcal{H}^{n}<\frac{\alpha_{1}}{4} .
$$

Observe that $\rho \Gamma^{s}$ is the normal graph of the function $\operatorname{sef}\left(\rho^{-1}\right.$.) on $\rho \Sigma$. As $\rho \Sigma \rightarrow \mathcal{C}(\Sigma)$ in $C_{\text {loc }}^{\infty}\left(\mathbb{R}^{n+1} \backslash\{\mathbf{0}\}\right)$ as $\rho \rightarrow 0$ and the link $\mathcal{L}(\Sigma)$ is smooth embedded, it follows from 4.2 and (4.3) that there is a $C_{A}\left(r_{1}\right)$ so that

$$
\sup _{|s| \leq \epsilon_{1}, \rho<1} \sup _{\rho \Gamma^{s} \backslash B_{r_{1}}}\left|A_{\rho \Gamma^{s}}\right|<C_{A}\left(r_{1}\right) .
$$

Furthermore, together with (C.14, (C.15) and (C.17), we get that, if $\mathbf{y}_{0} \in \mathbb{R}^{n+1}, \rho<1$, and $|s| \leq \epsilon_{1}$, then

$$
\begin{aligned}
F\left[\rho \Gamma^{s}-\mathbf{y}_{0}\right] & \leq F\left[\rho \Sigma-\mathbf{y}_{0}\right]+\frac{\alpha_{1}}{2}+(4 \pi)^{-\frac{n}{2}} \int_{\rho \Gamma^{s} \cap\left(B_{R}\left(\mathbf{y}_{0}\right) \backslash B_{r_{1}}\right)} e^{-\frac{\left|\mathbf{x}-\mathbf{y}_{0}\right|^{2}}{4}} d \mathcal{H}^{n} \\
& -(4 \pi)^{-\frac{n}{2}} \int_{\rho \Sigma \cap\left(B_{R}\left(\mathbf{y}_{0}\right) \backslash B_{r_{1}}\right)} e^{-\frac{\left|\mathbf{x}-\mathbf{y}_{0}\right|^{2}}{4}} d \mathcal{H}^{n} \\
& \leq F\left[\rho \Sigma-\mathbf{y}_{0}\right]+\frac{\alpha_{1}}{2}+C_{E},
\end{aligned}
$$

where $C_{E}$ depends on $C_{0}, C_{1}, \epsilon_{1}, r_{1}, C_{M}$, and $C_{A}$ with $C_{E} \rightarrow 0$ as $\epsilon_{1} \rightarrow 0$.

Now let us choose $\alpha_{1}=\min \left\{\alpha_{0} / 4,2(\lambda[\Sigma]-1) / 5\right\}$ and possibly shrink $\epsilon_{1}$ so that $C_{E}<\alpha_{0} / 4$. Hence, by [C.9], we conclude that for all $\left|\mathbf{x}_{0}\right|>\bar{R}^{\prime}=\max \left\{R^{\prime}, \frac{\epsilon_{3}}{2}\right\}$

$$
G\left(\mathbf{x}_{0}, t_{0}, s\right)<\lambda[\Sigma]-\frac{\alpha_{0}}{2}
$$

Finally, in view of (C.19), we only need to deal with the case that $t_{0}$ approaches to 0 and $\left|\mathbf{x}_{0}\right| \leq \bar{R}^{\prime}$ so to complete the proof of Item (5). We choose $\bar{t}$ sufficiently small so that

$$
e^{\frac{C_{H} \bar{t}}{4}}\left(\lambda[\Sigma]-\alpha_{0}\right)<\lambda[\Sigma]-\frac{\alpha_{0}}{2} .
$$

Thus, further shrinking $\epsilon_{1}$ if necessary, it follows from (C.13), (C.9) and Item (3) that for all $0<t_{0}<\bar{t},\left|\mathbf{x}_{0}\right| \leq \bar{R}^{\prime}$, and $|s| \leq \epsilon_{1}$, we have that $G\left(\mathbf{x}_{0}, t_{0}, s\right)<\lambda[\Sigma]-\alpha_{0} / 4$.

Acknowledgements. We would like to thank Detang Zhou for many constructive comments, in particular for explaining to us an alternative proof of the half-space theorem for self-shrinkers and the Rellich-Kondrachov type theorem of weighted Sobolev spaces. 


\section{REFERENCES}

[1] J. Bernstein and L. Wang, A remark on a uniqueness property of high multiplicity tangent flows in dimension three, Int. Math. Res. Not. IMRN 2015, no. 15, 6286-6294.

[2] J. Bernstein and L. Wang, A sharp lower bound for the entropy of closed hypersurfaces up to dimension six, preprint. Available at: http://arxiv.org/abs/1406.2966

[3] K. Brakke, The motion of a surface by its mean curvature, Mathematical Notes 20, Princeton University Press, Princeton, N.J., 1978.

[4] S. Brendle, Embedded self-similar shrinkers of genus 0, Ann. of Math. (2) 183, (2016), no. 2, 715-728.

[5] M. Cavalcante and J. Espinar, Halfspace type theorems for self-shrinkers, to appear in Bull. London Math. Soc.. Available at: http://arxiv.org/abs/1412.3754

[6] B. L. Chen and L. Yin, Uniqueness and pseudolocality theorems of the mean curvature flow, Comm. Anal. Geom. 15 (2007), no. 3, 435-490.

[7] X. Cheng and D. Zhou, Eigenvalues of the drifted Laplacian on complete metric measure spaces, to appear in Commun. Contemp. Math.. Available at:http://arxiv.org/abs/1305.4116

[8] T.H. Colding, T. Ilmanen, and W.P. Minicozzi II, Rigidity of generic singularities of mean curvature flow, Publ. Math. Inst. Hautes Études Sci. 121 (2015), no. 1, 363-382.

[9] T.H. Colding, T. Ilmanen, W.P. Minicozzi II, and B. White, The round sphere minimizes entropy among closed self-shrinkers, J. Differential Geom. 95 (2013), no. 1, 53-69.

[10] T. H. Colding and W.P. Minicozzi II, Generic mean curvature flow I; generic singularities, Ann. of Math. (2) 175 (2012), no. 2, 755-833.

[11] K. Ecker, Regularity theory for mean curvature flow, Progress in Nonlinear Differential Equations and their Applications, 57, Birkhäuser Boston, Inc., Boston, MA, 2004.

[12] K. Ecker and G. Huisken, Mean curvature evolution of entire graphs, Ann. of Math. (2) 130 (1989), no. 3, 453-471.

[13] K. Ecker and G. Huisken, Interior estimates for hypersurfaces moving by mean curvature, Invent. Math. 105 (1991), no. 3, 547-569.

[14] D. Gilbarg and N. Trudinger, Elliptic partial differential equations of second order, Second edition, Grundlehren der Mathematischen Wissenschaften [Fundamental Principles of Mathematical Sciences], 224, Springer-Verlag, Berlin,1983.

[15] G. Huisken, Local and global behaviour of hypersurfaces moving by mean curvature, Differential geometry: partial differential equations on manifolds (Los Angeles, CA, 1990), 175-191, Proc. Sympos. Pure Math., 54, Part 1, Amer. Math. Soc., Providence, RI, 1993.

[16] G. Huisken, Asymptotic behaviour for singularities of the mean curvature flow, J. Differential Geom. 31 (1990), no. 1, 285-299.

[17] T. Ilmanen, Singularities of mean curvature flow of surfaces, preprint. Available at: http://www . math . ethz.ch/ ilmanen/papers/pub.html

[18] T. Ilmanen, A. Neves, and F. Schulze, On short time existence for the planar network flow, preprint. Available at: http://arxiv.org/abs/1407.4756

[19] G.M. Lieberman, Second order parabolic differential equations, World Scientific Publishing Co., Inc., River Edge, NJ, 1996.

[20] H. Samelson, Orientability of hypersurfaces in $\mathbb{R}^{n}$, Proc. Amer. Math. Soc. 22 (1969), 301-302.

[21] N. Sesum, Rate of convergence of the mean curvature flow, Comm. Pure Appl. Math. 61 (2008), no. 4, 464-485.

[22] L. Simon, Existence of surfaces minimizing the Willmore functional, Comm. Anal. Geom. 1 (1993), no. 2, 281-326.

[23] K. Smoczyk, Starshaped hypersurfaces and the mean curvature flow, Manuscripta Math. 95 (1998), no. 2, 225-236.

[24] A. Stone, A density function and the structure of singularities of the mean curvature flow, Calc. Var. Partial Differential Equations 2 (1994), no. 4, 443-480.

[25] L. Wang, Uniqueness of self-similar shrinkers with asymptotically conical ends, J. Amer. Math. Soc. 27 (2014), no. 3, 613-638.

[26] B. White, A local regularity theorem for mean curvature flow, Ann. of Math. (2) 161 (2005), no. 3, 14871519. 
Department of Mathematics, Johns Hopkins University, 3400 N. Charles Street, BaltiMORE, MD 21218

E-mail address: bernstein@math. jhu.edu

Department of MAThematics, University of Wisconsin-MADison, 480 LinCOLn DRIVE, MADISON, WI 53706

E-mail address: luwang@math.wisc.edu 